\title{
Renormalization-Group Analysis of the Generalized sine-Gordon Model and of the Coulomb Gas for $d \geq 3$ Dimensions
}

\author{
I. Nándori ${ }^{1,2,3}$, U. D. Jentschura ${ }^{1}$, K. Sailer ${ }^{2}$, and G. Soff ${ }^{1}$ \\ 1 Institut für Theoretische Physik, Technische Universität Dresden, 01062 Dresden, Germany \\ 2 Department of Theoretical Physics, University of Debrecen, H-4032, Debrecen, Hungary and \\ 3 Institute of Nuclear Research, P.O.Box 51, H-4001 Debrecen, Hungary
}

(Dated: June 19, 2018)

\begin{abstract}
Renormalization-group ( $R G$ ) flow equations have been derived for the generalized sine-Gordon model (GSGM) and the Coulomb gas (CG) in $d \geq 3$ of dimensions by means of Wegner's and Houghton's, and by way of the real-space RG approaches. The UV scaling laws determined by the leading-order terms of the flow equations are in qualitative agreement for all dimensions $d \geq$ 3 , independent of the dimensionality, and in sharp contrast to the special case $d=2$. For the 4-dimensional GSGM it is demonstrated explicitly (by numerical calculations), that the blocked potential tends to a constant effective potential in the infrared (IR) limit, satisfying the requirements of periodicity and convexity. The comparison of the RG flows for the three-dimensional GSGM, the $\mathrm{CG}$, and the vortex-loop gas reveals a significant dependence on the renormalization schemes and the approximations used.
\end{abstract}

PACS numbers: 11.10.Hi, 11.10.Kk

\section{INTRODUCTION}

The renormalization of a two-dimensional one-component scalar field theory with a periodic self-interaction, and the two-dimensional generalized sine-Gordon model (GSGM) have been investigated for $d=2$ dimensions in great detail over the last three decades 1 , 2, 3, 3, , 5, 6, 7, 8, 9, 10, 11, 12, 13, 14, 15. Here, we only mention some of the main results:

- The blocked potential for the two-dimensional GSGM tends to a constant effective potential in the IR limit, independent of the field [12, 13, 14].

- Following the procedure proposed in [16], one can identically rewrite the partition function of the $d$-dimensional GSGM in the form of a $d$-dimensional static Coulomb gas, which we call here the equivalent Coulomb gas (ECG). For $d=2$ it is well-known that the GSGM and the ECG, as well as the XY model describing classical planar spins belong to the same universality class [10]. The XY model has a dual theory in the sense of [17] that can be reformulated as a gas of topological excitations: the two-dimensional vortex gas (VG) for $d=2$, and the three-dimensional vortex-loop gas (VLG) for $d=3$ (see [10] and Refs. therein). For dimensions $d=2$, it was shown that the ECG and the VG can be transformed into one another by an appropriate duality transformation 3, 7, 8, 15 that inverts the temperature. Two-dimensional generalized models are well known where both the ECG and the VG are included as particular limiting cases [3, 7, 8, 15] and are self-dual under the duality transformation.

While the relations of the various models for two dimensions are well-established, those of the corresponding models for $d=3$ are not completely settled (see e.g. 10]). In particular, it is not proven that the VLG and the threedimensional XY-model belong to the same class of universality. Therefore, the investigation of the renormalization of the GSGM and the ECG for $d \geq 3$ dimensions is essential for a further clarification of this issue. The purpose of the present work is threefold:

1. to investigate the renormalization of the GSGM for dimensions $d \geq 2$ by the Wegner-Houghton renormalizationgroup (WH-RG) method [18] and to demonstrate numerically that the blocked potential for the 4-dimensional GSGM tends to a constant effective potential in the IR limit; and

2. to perform an RG analysis for the ECG for dimensions $d \geq 2$ by the real-space renormalization group (RS-RG) method using the dilute gas approximation (DGA) [7]; and finally

3. to compare the RG flows obtained for the GSGM, ECG and VLG 19, 20, in the three-dimensional case.

Renormalization of the GSGM and that of the related models in $d \geq 3$ dimensions are of particular interest with regard to the numerous physical applications. In high-energy physics the haaron model [16] of confinement is in need of a clarification of the IR behaviour of the GSGM in $d=4$ dimensions. In low-temperature physics the superfluid 
transition of $\mathrm{He}^{4}$ belongs to the universality class of the three-dimensional XY model and it provides an exceptional opportunity for an experimental test of the RG predictions [21]. In superconductivity the three-dimensional XY model (isotropic and anisotropic) was used to study the phase transitions in the three-dimensional flux-line lattice 22, 23]. The three-dimensional XY model can also help us in understanding the flux motions in superconductors with extremely high anisotropy; these have been observed in recent experiments [24].

Due to the fact that the GSGM and the ECG are equivalent at the level of the partition function, their renormalization-group flows obtained by applying different RG methods are alternative approaches to tackle essentially the same problem. On the one hand, the WH-RG applied to the GSGM keeps the periodicity of the self-interaction potential in each infinitesimal blocking step. There is a price to pay: one has to handle infinitely many vertices in the process of searching the blocked action in the subspace of functionals periodic in the field variable. Furthermore, the requirements of periodicity and convexity 25] are laid upon the effective potential of the model which constrain it to a field-independent constant [12]. The WH-RG has the advantage that (i) it can be extrapolated to the IR limit and (ii) is able to treat the spinodal instability [26]. Its main disadvantage is that the truncation of the gradient expansion is unavoidable and that the implementation of the sharp momentum cut-off can induce unphysical features of the RG flow going beyond the local-potential approximation (LPA) 27]. By restricting ourselves to LPA, we ignore the wave-function renormalization, which is equivalent to neglecting the scale-dependence of the temperature beyond the tree-level and its physical consequences.

On the other hand, the RS-RG applied to the ECG keeps intact the CG structure in each blocking step and therefore also the periodicity, albeit indirectly. It implements a sharp cut-off in real space and has been proven very adequate for applications to the two-dimensional Coulomb gas [7] and to the three-dimensional vortex-loop gas [19, 20]. Nevertheless, it has the drawbacks that (i) no systematic approach is known to go beyond the dilute-gas approximation (DGA), and (ii) the sharp cut-off in real space, which is effectively a smooth momentum cut-off, is inadequate to treat the spinodal instability. Because our WH-RG approach will show that the latter always occurs, the interpretation of the extrapolation of our RS-RG results for the ECG to the IR regime is problematic. Nevertheless, it is interesting to compare the RS-RG results for the ECG in the UV regime to those for the gas of topological excitations (e.g. the VLG for $d=3$ ).

The phase transition of systems in the universality class of the two-dimensional Coulomb gas, known as the Kosterlitz-Thouless-Berezinskii phase transition, is a topological phase transition because there is no spatial longrange order for $d=2$ dimensions at finite temperature due to the Mermin-Wagner theorem [28]. For $d \geq 3$ dimensions the Mermin-Wagner theorem does not hold any more. Therefore, one expects a modification of the phase structure of the Coulomb gas in the number of dimensions $d>2$ as compared to the case with $d=2$. As shown below, the UV scaling laws in $d>2$ for both the GSGM and the ECG share the property of a lacking Coleman fixed point, which by contrast is present for $d=2[2]$.

In Sect. [I] we introduce our notations and summarize RG flow equations obtained in the literature for the various models discussed in the next sections. The RG flow and the blocked potential for the 4-dimensional GSGM are determined numerically in Sect. [II] by the WH-RG method. In Sect. IV the RG flow obtained by the RS-RG approach for the $d$-dimensional Coulomb gas is discussed. In Sect. $\nabla$ the RG flow of the three-dimensional Coulomb gas (ECG) is compared to the RG flow obtained in for the three-dimensional GSGM, and to those for the threedimensional VLG 20]. Finally, the results are summarized in Sect. VI] RS-RG flow equations for the $d$-dimensional Coulomb gas are derived in App. \$

\section{GSGM AND RELATED MODELS}

\section{A. GSGM and ECG}

First, we remind the reader that the partition function for the GSGM can be identically rewritten in the form of the partition function for a CG, that we shall call the equivalent CG (ECG). In its lattice regulated form the partition function of the GSGM is given as

$$
Z_{\mathrm{GSGM}}=\int \mathcal{D}[\phi] \exp \left[-\frac{1}{2} a^{2 d} \sum_{x, y} \phi_{x} d_{x, y} \phi_{y}-a^{d} \sum_{x} \sum_{n=1}^{\infty} u_{n} \cos \left(n \beta \phi_{x}\right)\right],
$$

where $a$ denotes the lattice spacing, and $d_{x, y}=2 a^{-d-2}\left(d \delta_{x, y}-\sum_{\mu=1}^{d} \delta_{y, x+n_{\mu} a}\right)$ is the inverse propagator. Performing the transformation of the field variable $\varphi_{x}=\beta \phi_{x}$, one finds

$$
Z=\int \mathcal{D}[\varphi] \exp \left[-\frac{a^{2 d}}{2 \beta^{2}} \sum_{x, y} \varphi_{x} d_{x, y} \varphi_{y}-a^{d} \sum_{x} \sum_{n=1}^{\infty} u_{n} \cos \left(n \varphi_{x}\right)\right] .
$$


This partition function can be identically rewritten in the form of the macrocanonical partition function for a CG applying the procedure proposed in [16]:

1. One expands the exponential factor of the integrand with the periodic potential in Taylor series, expresses $\cos (n \varphi)$ in terms of the exponential function, and introduces the integer valued variable, the charge $\sigma=$ $\pm 1, \pm 2, \ldots$; the result of these operations is

$$
Z=\left(\prod_{x} \int d \varphi_{x}\right) \sum_{\nu=0}^{\infty} \frac{(-1)^{\nu}}{2^{\nu} \nu !} a^{\nu d} \sum_{\substack{x_{1}, x_{2}, \ldots, x_{\nu} \\ \sigma_{1}, \ldots, \sigma_{\nu} \neq 0}} u_{\sigma_{1}} \cdots u_{\sigma_{\nu}} \exp \left[-\frac{a^{2 d}}{2 \beta^{2}} \sum_{x, y} \varphi_{x} d_{x, y} \varphi_{y}+i a^{d} \sum_{x} \rho_{x} \varphi_{x}\right]
$$

where the charge density, $\rho_{x}=\sum_{j=1}^{\nu} \sigma_{j} a^{-d} \delta_{x, x_{j}}$, has been introduced and $u_{-\sigma}=u_{\sigma}$ holds due to the $Z(2)$ symmetry of the potential.

2. Performing the Gaussian path integral, one obtains

$$
\begin{aligned}
Z & =\sum_{\nu=0}^{\infty} \frac{(-1)^{\nu}}{2^{\nu} \nu !} a^{\nu d} \sum_{\substack{x_{1}, x_{2}, \ldots, x_{\nu} \\
\sigma_{1}, \ldots, \sigma_{\nu} \neq 0}} u_{\sigma_{1}} \cdots u_{\sigma_{\nu}} I_{x_{1}, \ldots, x_{\nu}}^{\sigma_{1}, \ldots, \sigma_{\nu}}, \\
I_{x_{1}, \ldots, x_{\nu}}^{\sigma_{1}, \ldots, \sigma_{\nu}} & =\exp \left[-\frac{1}{2} \operatorname{Tr} \ln G^{-1}-\frac{1}{2} \beta^{2} \sum_{i, j=1}^{\nu} \sigma_{i} \sigma_{j} G_{x_{i}, x_{j}}\right]
\end{aligned}
$$

with the (dimensionful) propagator $G_{x, y}=a^{-2 d}\left(d^{-1}\right)_{x, y}$.

Including the self-interaction terms into the (dimensionless) fugacities, $w_{\sigma}=-\frac{1}{2} a^{d} u_{\sigma} \exp \left[-\frac{1}{2} \sigma^{2} \beta^{2} G_{x_{i}, x_{i}}\right]$ and replacing $G_{x, x}$ by the value $G(a)$ of the propagator at the cut-off distance $a$, the partition function (4) takes the form

$$
Z_{\mathrm{ECG}} \equiv \sum_{\nu=0}^{\infty} Z^{[\nu]}=\sum_{\nu=0}^{\infty} \frac{1}{\nu !} \int_{D_{0}} \frac{d^{d} x_{1}}{a^{d}} \int_{D_{1}} \frac{d^{d} x_{2}}{a^{d}} \cdots \int_{D_{\nu-1}} \frac{d^{d} x_{\nu}}{a^{d}} \sum_{\sigma_{1}, \ldots, \sigma_{\nu} \neq 0} w_{\sigma_{1}} \cdots w_{\sigma_{\nu}} J_{x_{1}, \ldots, x_{\nu}}^{\sigma_{1}, \ldots, \sigma_{\nu}}
$$

where

$$
\begin{aligned}
J_{x_{1}, \ldots, x_{\nu}}^{\sigma_{1}, \ldots, \sigma_{\nu}} & =\exp \left[-\frac{1}{2} \operatorname{Tr} \ln \tilde{G}^{-1}-\frac{1}{2} \tilde{\beta}^{2} \sum_{i \neq j}^{\nu} \sigma_{i} \sigma_{j} \tilde{G}_{x_{i}, x_{j}}\right] \\
w_{\sigma} & =-\frac{1}{2} a^{d} u_{\sigma} \exp \left[-\frac{1}{2} \sigma^{2} \tilde{\beta}^{2} \tilde{G}(a)\right]
\end{aligned}
$$

with the dimensionless coupling $\tilde{\beta}^{2}=a^{-(d-2)} \beta^{2}$ and the dimensionless propagator

$$
\tilde{G}_{x, y}= \begin{cases}-c_{2} \ln \left(\frac{|x-y|}{\bar{a}}\right) & \text { for } \quad d=2, \\ c_{d} \frac{\bar{a}^{d-2}}{(d-2)|x-y|^{d-2}} & \text { for } \quad d \geq 3 .\end{cases}
$$

Here, $c_{d}=\Omega_{d}^{-1}$ where $\Omega_{d}$ denotes the entire solid angle in $d$ dimensions, and $\bar{a}=\mathcal{O}(a)$. Its explicit value has been determined for $d=2$ (c.f. 10$]$ ). For the sake of simplicity we shall take here $\bar{a}=a$. The integration regions on the r.h.s. of Equation (5) are discussed in App. A Eq. (5) represents the grand-canonical partition function for a gas of static Coulomb charges. The temperature of this ECG is given as $\propto\left(\beta^{2}\right)^{-1}$, and the Fourier amplitudes $u_{\sigma}$ of the periodic self-interaction potential in the GSGM play the role of the fugacities of the various charges $\sigma= \pm 1, \pm 2, \ldots$; this mapping of the partition function of the GSGM to that of the ECG is exact for arbitrary dimensionality.

In this work we shall check our RS-RG results for the ECG in higher dimensions against the RG-flow equations obtained in 7] with the same approach for $d=2$. It is a little cumbersome to identify the various notations (see Tab. [for an explanation). Indeed, the two-dimensional ECG is an electric Coulomb gas in the sense of 7]. Applying 
the RG flow equations [7, Eqs. (2.22) and (2.23)] to the two-dimensional ECG with $Z(2)$ symmetry $\left(w_{\sigma}=w_{-\sigma}\right)$, one obtains:

$$
a \frac{d \tilde{\beta}^{2}}{d a}=-\pi \tilde{\beta}^{4} \sum_{\sigma=1}^{\infty} \sigma^{2} w_{\sigma}^{2}, \quad a \frac{d w_{\sigma}}{d a}=\left(2-\frac{\tilde{\beta}^{2}}{4 \pi} \sigma^{2}\right) w_{\sigma}+\pi \sum_{\sigma^{\prime} \neq 0, \sigma} w_{\sigma^{\prime}} w_{\sigma-\sigma^{\prime}}-\pi\left(\frac{8 \pi}{3}+\sqrt{3}\right) w_{\sigma} \sum_{\sigma^{\prime}=1}^{\infty} w_{\sigma^{\prime}}^{2} .
$$

From the linearized flow equations one recovers the Coleman fixed point [2] of the sine-Gordon model at $\tilde{\beta}^{2}=8 \pi$. Below, we also compare our RS-RG flow equations derived for the ECG for arbitrary dimensionality to Eq. (8).

\begin{tabular}{|c|c||c|c|}
\hline$[7]$ & $\mathrm{ECG}$ & {$[7]$} & $\mathrm{VG}$ \\
\hline $1 / g$ & $\tilde{\beta}^{2} /(2 \pi)$ & $g$ & $2 \pi J$ \\
$e$ & $\sigma$ & $m$ & $q$ \\
$Y(e, 0)$ & $w_{\sigma}$ & $Y(0, m)$ & $A_{q}$ \\
\hline
\end{tabular}

TABLE I: Identification of the various notations for the parameters of the two-dimensional ECG (first and second columns), as well as for the VG (third and fourth columns) according to Eqs. (2.1) and (2.2) in [7] and our Eqs. (2), (5), and (10).

\section{B. Gas of topological excitations}

In general, dual transformations [17] can be found and applied to almost any Abelian theory in any number of dimensions. The dual form of the original theory represents an intermediate step towards its third form when the partition function is expressed in terms of the topological excitations of the original degrees of freedom (see [10]), the gas of topological excitations.

The $d$-dimensional XY-model is given by the partition function

$$
Z_{X Y}=\int \mathcal{D}\left[\vec{S}_{x}\right] \delta\left(\vec{S}_{x}^{2}-1\right) \exp \left[-\sum_{<y, z>}(-J) \vec{S}_{y} \cdot \vec{S}_{z}\right]
$$

where the classical spin $\vec{S}_{x}$ is a unit-vector in the two-dimensional internal space; $\sum_{<y, z>}$ stands for the sum over pairs of nearest neighbour lattice sites. For the numbers $d=2$ and $d=3$ of dimensions the corresponding gases of topological excitations are well-known:

1. The vortex-gas (VG) for $d=2$ dimensions. The partition function of the XY-model can be rewritten as (see [10])

$$
Z_{V G}=\sum_{\nu=0}^{\infty} \frac{1}{\nu !} \sum_{x_{1}, \ldots, x_{\nu}} A_{q_{1}} \cdots A_{q_{\nu}} \exp \left[-\frac{1}{2} 4 \pi^{2} J \sum_{i \neq j}^{\nu} q_{i} q_{j} \tilde{G}_{x_{i}, x_{j}}\right] .
$$

This describes a system of static vortex charges interacting via the two-dimensional Coulomb potential

$$
\tilde{G}_{x_{i}, x_{j}}=-\frac{1}{2 \pi} \ln \left(\left|x_{i}-x_{j}\right| / a\right),
$$

where the fugacities of the vortices are defined as $A_{q}=\exp \left[-\mu q^{2} J / 2\right] ; q= \pm 1, \pm, 2, \ldots$, and $\mu$ can be interpreted as the chemical potential of the vortex-antivortex pairs. The VG and the ECG are both Coulomb gases, but those of different physical origin. The vortices are the topological excitations of the free periodic scalar field, 
whereas the Coulomb charges of the ECG are connected with the periodic self-interaction of the scalar field. Using the identification of various notations given in Table (last two columns), one may interpret the VG as a magnetic Coulomb gas in the sense of [7]. Again, applying the RG flow equations 7, Eqs. (2.22) and (2.23)] for the VG with $Z(2)$ symmetry $\left(A_{q}=A_{-q}\right)$, one finds

$$
a \frac{d J}{d a}=-4 \pi^{3} J^{2} \sum_{q=1}^{\infty} q^{2} A_{q}^{2}, \quad a \frac{d A_{q}}{d a}=\left(2-\pi J q^{2}\right) A_{q}+\pi \sum_{q^{\prime} \neq 0, q} A_{q^{\prime}} A_{q-q^{\prime}}-\pi\left(\frac{8 \pi}{3}+\sqrt{3}\right) A_{q} \sum_{q^{\prime}=1}^{\infty} A_{q^{\prime}}^{2}
$$

According to Table the RG equations (11) can be rewritten in terms of $\tilde{\beta}^{2}=1 / J$ as

$$
a \frac{d \tilde{\beta}^{2}}{d a}=4 \pi^{3} \sum_{q=1}^{\infty} q^{2} A_{q}^{2}, \quad a \frac{d A_{q}}{d a}=\left(2-\frac{\pi}{\tilde{\beta}^{2}} q^{2}\right) A_{q}+\pi \sum_{q^{\prime} \neq 0, q} A_{q^{\prime}} A_{q-q^{\prime}}-\pi\left(\frac{8 \pi}{3}+\sqrt{3}\right) A_{q} \sum_{q^{\prime}=1}^{\infty} A_{q^{\prime}}^{2}
$$

2. The vortex-loop gas ( $V L G$ ) for $d=3$ dimensions. For the three-dimensional XY model the dual theory has been constructed in the literature [19, 20] and it turned out to be the gas of interacting vortex loops. Also the corresponding flow equations have been derived for the parameters $K \propto J$ and $y$ (the fugacity of the vortex loops) by RS-RG in the DGA:

$$
a \frac{d K}{d a}=K-\frac{4 \pi^{3}}{3} K^{2} y, \quad a \frac{d y}{d a}=\left(6-\pi^{2} K L\right) y
$$

where $L \equiv L(a)$ corresponds to the long-range contribution. One may assume $L$ to approach a constant in the IR limit, or to be weakly (logarithmically) divergent, e.g. $L(a)=1$ in Ref. [19], $L(a)=\ln \left(a / a_{c}\right)+1$ in Ref. [20].

\section{RG FLOW OF GSGM}

\section{A. Differential RG in momentum space}

Below we shall discuss the renormalization of the GSGM (Euclidean one-component scalar field theory with periodic self-interaction) by means of the differential renormalization group approach in momentum space. In this approach, blocking transformations 29] are realized by successive elimination of the field fluctuations according to their decreasing momentum in infinitesimal steps. The physical effects of the eliminated modes are encoded in the dependence of the coupling constants on the scale $k$ above which the high-frequency modes are effectively eliminated. The elimination of the modes above the moving scale $k$ is either complete as in Wegner's and Houghton's method (WH-RG) [18] (sharp IR cut-off) or partial as in Polchinski's method 30] (smooth IR cut-off). Both of these RG methods keep intact the periodicity, which is the essential symmetry of theory. Nevertheless, choosing the WH-RG method is a necessity in our case: as shown below, a spinodal instability occurs at some critical momentum scale $k_{c}$. Therefore, the elimination of the modes above and below this critical scale involves completely different physics. Renormalization of the couplings occurs due to small quantum fluctuations above the critical scale, whereas tree-level fluctuations of large amplitude dominate below that scale [26]. Thus, the application of the RG methods with smooth cut-off like that of Polchinski's method [30, 31, 32] is now unjustified, because such methods treat the modes at all scales similarly.

Above the critical scale $k_{c}$ the WH-RG method [18] provides an integro-differential equation for the blocked action $S_{k}[\phi]$ as the functional of the scalar field $\phi$. In order to solve this equation, one has to project it to a particular functional subspace. Therefore, one generally assumes that the blocked action contains only local interactions, expands it in powers of the gradient of the field, and truncates this expansion at a given order, for technical reasons. The drawback of the WH-RG method combined with the gradient expansion is that the truncation of the latter may introduce an unphysical high-frequency behaviour of the blocked action, since a sharp cut-off is used [27]. Here, we restrict ourselves to the leading order of the gradient expansion, the local-potential approximation (LPA). In order to get more insight in the RG evolution of the blocked action, it may turn out to be useful in the future to replace the gradient expansion with some more appropriate approximation scheme, like the cluster expansion proposed in [33]. In LPA the ansatz for the blocked action can be written as

$$
S_{k}=\int \mathrm{d}^{d} x\left[\frac{1}{2}\left(\partial_{\mu} \phi\right)^{2}+V_{k}(\phi)\right]
$$


with the blocked potential $V_{k}(\phi)$ for which the WH equation reads as follows [34, 35, 36, 37, 38]:

$$
k \partial_{k} V_{k}(\phi)=-k^{d} \alpha_{d} \ln \left(\frac{k^{2}+\partial_{\phi}^{2} V_{k}(\phi)}{k^{2}}\right),
$$

with $\alpha_{d}=\frac{1}{2} \Omega_{d}(2 \pi)^{-d}$, where $\Omega_{d}$ denotes the solid angle in $d$ dimensions. In order to look for fixed-point solutions of (15) or to follow the scaling of an arbitrary potential, it is convenient to remove the trivial scaling of the dimensionful coupling constants and rewrite Eq. (15) as

$$
\left(d-\frac{d-2}{2} \tilde{\phi} \partial_{\tilde{\phi}}+k \partial_{k}\right) \tilde{V}_{k}(\tilde{\phi})=-\hbar \alpha_{d} \ln \left(1+\partial_{\tilde{\phi}}^{2} \tilde{V}_{k}(\tilde{\phi})\right)
$$

where the dimensionless quantities $\tilde{\phi}=k^{-\frac{d-2}{2}} \phi$ and $\tilde{V}_{k}(\tilde{\phi})=k^{-d} V_{k}(\phi)$ have been introduced.

Notice that the argument of the logarithm in Eqs. (15) and (16) must be positive. If the argument vanishes or if it changes sign at a critical value $k_{\mathrm{c}}$, given by $k_{\mathrm{c}}^{2}=-\partial_{\phi}^{2} V_{k_{\mathrm{c}}}(\phi)$, the WH equation (15) loses its validity for $k<k_{\mathrm{c}}$. This is a consequence of the spinodal instability. At the scale $k_{\mathrm{c}}$ zeros occur among the eigenvalues of the second derivative matrix of the blocked action, the restoring force vanishes for some modes of the fluctuations and the amplitudes of such fluctuations can grow to a large finite value and cause a tree-level renormalization [26]. Then, the tree-level blocking relation [26]

$$
V_{k-\delta k}(\phi)=\min _{\rho}\left[k^{2} \rho^{2}+\frac{1}{2} \int_{-1}^{1} \mathrm{~d} u V_{k}(\phi+2 \rho \cos (\pi u))\right],
$$

should be used instead of Eq. (15) for the determination of the blocked action $V_{k-\delta k}(\phi)$ at the lower scale $k-\delta k$.

\section{B. Global aspects of the renormalization of the GSGM}

As it is argued in Refs. 12, 14 for $d=2$ dimensions, the WH-RG procedure retains the periodicity of the potential. The argumentation holds for any number of dimensions: both Eqs. (16) and (17) which describe the infinitesimal blocking step $\delta k$ can be considered in the form $V_{k-\delta k}(\phi)=\mathcal{F}\left[V_{k}(\phi)\right]$ with some functional $\mathcal{F}$. If the r.h.s. of this equation is periodic at the scale $k$ due to $V_{k}(\phi)=V_{k}(\phi+\Delta)$, then its l.h.s., i.e. the blocked potential $V_{k-\delta k}(\phi)$, should also be periodic with the same period $\Delta$. This means that (i) the WH-RG in LPA keeps periodicity of the blocked potential, and (ii) the period $\Delta$ in the internal space does not depend on the scale. Note that these statements are only valid for the dimensionful potential as the local functional of the dimensionful field.

Since the blocked potential $V_{k}(\phi)$ tends to the effective potential $V_{\text {eff }}(\phi)$ in the IR limit $k \rightarrow 0$, the effective potential should be periodic, as well. On the other hand, the effective potential is convex [25] over the homogeneous field configurations. The double requirement of periodicity and convexity can only be fulfilled in a trivial manner, namely if $V_{\text {eff }}(\phi)$ is a constant function. In order to demonstrate this general statement, we determine the blocked potential for the 4-dimensional GSGM numerically.

\section{RG equations}

Here, we apply the flow equations (16) and (17) for the GSGM. For the sake of simplicity, we consider periodic potentials with $\mathrm{Z}(2)$ symmetry, $V_{k}(\phi)=V_{k}(-\phi)$. The Fourier-expansion of such potentials is given by

$$
V_{k}(\phi)=\sum_{n=1}^{\infty} u_{n}(k) \cos (n \beta \phi), \quad \tilde{V}_{k}(\tilde{\phi})=\sum_{n=1}^{\infty} \tilde{u}_{n}(k) \cos (n \tilde{\beta} \tilde{\phi})
$$

where $\beta=2 \pi / \Delta$ is independent of the scale $k$, and the tilde indicates the corresponding dimensionless quantities.

Inserting the Fourier-expansion (18) into Eq. (16) differentiated with respect to $\tilde{\phi}$, one obtains an equation with terms either non-periodic or periodic in the field variable $\phi$. The non-periodic terms occur due to the scale-dependence of $\tilde{\beta}(k)$ and the term $-\frac{d-2}{2} \tilde{\phi} \partial_{\tilde{\phi}} \tilde{V}_{k}(\tilde{\phi})$ in Eq. (16). The obtained flow equation is satisfied if and only if the non-periodic and the periodic parts are identical on both sides separately. The non-periodic parts yield:

$$
\sum_{n} \tilde{u}_{n}(k) n\left(k \partial_{k} \tilde{\beta}(k)-\frac{d-2}{2} \tilde{\beta}(k)\right) \tilde{\phi} \sin (n \tilde{\beta} \tilde{\phi})=0 .
$$


Using this relation and the equation for the periodic terms, one gets the evolution equations for arbitrary number $d$ of dimensions as given previously in Ref. [12]:

$$
\begin{gathered}
\left(d+k \partial_{k}\right) v_{n}(k)=\alpha \tilde{\beta}^{2}(k) n^{2} v_{n}(k)-\frac{1}{2} \tilde{\beta}^{2}(k) \sum_{p=1}^{N} A_{n, p}(k)\left(d+k \partial_{k}\right) v_{p}(k), \\
k \partial_{k} \tilde{\beta}^{2}(k)=(d-2) \tilde{\beta}^{2}(k),
\end{gathered}
$$

where $v_{n}(k)=n \tilde{u}_{n}(k)$, and $A_{n, p}(k)=|n-p| v_{n-p}-(n+p) v_{n+p}$ [note that in our previous definitions [12] the analog of the first relation is $\left.v_{n}(k)=n \beta \tilde{u}_{n}(k)\right]$. Eq. (21) follows from Eq. (19) and has the solution

$$
\tilde{\beta}^{2}(k)=k^{d-2} \beta^{2} .
$$

This expresses that the periodicity of the blocked potential is kept, whereas the dimensionful period $\Delta=\frac{2 \pi}{\beta}$ remains independent of the scale.

In order to solve the flow equations (20), one has to express the derivatives $k \partial_{k} \tilde{u}_{n}(k)$ occurring in Eq. (20) explicitly and truncate the Fourier-expansion of the potential at some mode $n=N$. This can be achieved by the following steps:

1. Rewrite Eq. (20) in terms of $\tilde{u}_{n}=\frac{1}{n} v_{n}$ :

$$
\sum_{p=1}^{\infty}\left(\delta_{n, p}+\frac{1}{2} \tilde{\beta}^{2} \tilde{A}_{n, p}\right) k \partial_{k} \tilde{u}_{p}(k)=-d \tilde{u}_{n}(k)+\alpha_{d} \tilde{\beta}^{2}(k) n^{2} \tilde{u}_{n}(k)-\frac{d}{2} \tilde{\beta}^{2}(k) \sum_{p=1}^{\infty} \tilde{A}_{n, p}(k) \tilde{u}_{p}(k),
$$

with $\tilde{A}_{n, p}(k)=\frac{p}{n}\left[(n-p)^{2} \tilde{u}_{n-p}-(n+p)^{2} \tilde{u}_{n+p}\right]$.

2. Invert the matrix on the l.h.s. of Eq. (23) numerically in the subspace of the modes with $n, p \leq N$.

For later comparison with the flow equations for the $d$-dimensional ECG, it is useful to perform the inversion of the matrix $1+\frac{1}{2} \tilde{\beta}^{2} \tilde{A}$ keeping the terms up to the order $\mathcal{O}\left(\tilde{A}^{2}\right)$, i.e. those up to $\mathcal{O}\left(\tilde{u}^{3}\right)$ :

$$
k \partial_{k} \tilde{u}_{n}(k)=-\left[d-\alpha_{d} \tilde{\beta}^{2}(k) n^{2}\right] \tilde{u}_{n}(k)-\frac{\alpha_{d}}{2} \tilde{\beta}^{4} \sum_{p=1}^{\infty} \tilde{A}_{n, p} p^{2} \tilde{u}_{p}+\frac{\alpha_{d}}{4} \tilde{\beta}^{6} \sum_{p, r=1}^{\infty} \tilde{A}_{n, r} \tilde{A}_{r, p} p^{2} \tilde{u}_{p}+\mathcal{O}\left(\tilde{u}^{4}\right) .
$$

In the region of the spinodal instability for $k<k_{c}$, the RG flow should be determined with the help of the tree-level blocking relation (17) which can be rewritten for the dimensionful periodic potential as

$$
V_{k-\delta k}(\phi)=\min _{\rho}\left[k^{2} \rho^{2}+\sum_{n=0}^{\infty} u_{n}(k) \cos (n \beta \phi) J_{0}(2 n \beta \rho)\right]
$$

where $J_{0}$ stands for a Bessel function, $\beta=$ constant and $\phi$ is a homogeneous field configuration. It is worthwhile to note that Eq. (25) is just the same for any number $d$ of dimensions. Therefore, it can always be solved in the same manner as discussed in [12] for the case with $d=2$. The position $\rho=\rho_{k}(\phi)$ of the minimum should be determined separately for each constant field configuration $\phi$ at the given scale $k$. Then, the r.h.s. of Eq. (25) at the minimum as a function of $\phi$ should be reexpanded in Fourier-modes, in order to determine the coupling constants $u_{n}(k-\delta k)$ of the blocked potential at the lower scale $k-\delta k$. The pure tree-level scale-dependence (22) should hold in both regions $k>k_{\mathrm{c}}$ and $k<k_{\mathrm{c}}$ alike.

\section{UV scaling}

The trivial Gaussian fixed point $\tilde{V}^{*}=0$ is the only one of the Wegner-Houghton RG equation (16) in the subspace of periodic potentials. This is because the dimensionless coupling $\tilde{\beta}(k)$ can only be independent of the scale for a periodic potential that vanishes identically, as Eq. (22) should hold for a constant $\beta$.

In order to determine the scaling operators and the critical exponents of the periodic field theory around the Gaussian fixed point for $d \geq 3$ dimensions, one can use the quasi-linear version of the flow equation (24):

$$
\left(d+k \partial_{k}\right) \tilde{u}_{n}(k)=\alpha_{d} \tilde{\beta}^{2} n^{2} \tilde{u}_{n}(k) .
$$


Together with Eq. (22) this has the analytic solution

$$
\tilde{u}_{n}(k)=\tilde{u}_{n}(\Lambda)\left(\frac{k}{\Lambda}\right)^{-d} \exp \left\{\frac{\alpha_{d} \tilde{\beta}^{2}(\Lambda) n^{2}}{d-2}\left[\left(\frac{k}{\Lambda}\right)^{d-2}-1\right]\right\}
$$

where $\tilde{\beta}(\Lambda)$ and $\tilde{u}_{n}(\Lambda)$ are the bare values of the couplings. Let us consider the solution (27) in the neighbourhood of the UV cut-off $\Lambda$. Introducing a small positive parameter $\epsilon=1-\frac{k}{\Lambda}>0$, the term $\left(\frac{k}{\Lambda}\right)^{d-2}$ can be expanded around $\epsilon=0$ : $(1-\epsilon)^{d-2}=1-(d-2) \epsilon+\mathcal{O}\left(\epsilon^{2}\right)$. Inserting this into the exponent on the r.h.s. of Eq. (27) and using $\ln (1-\epsilon)=-\epsilon+\mathcal{O}\left(\epsilon^{2}\right)$, one finds

$$
\exp \left(-\alpha_{d} \tilde{\beta}^{2}(\Lambda) n^{2} \epsilon+\mathcal{O}\left(\epsilon^{2}\right)\right)=\exp \left(\alpha_{d} \tilde{\beta}^{2}(\Lambda) n^{2} \ln (1-\epsilon)+\mathcal{O}\left(\epsilon^{2}\right)\right) \approx(1-\epsilon)^{\alpha_{d} \tilde{\beta}^{2}(\Lambda) n^{2}} .
$$

Then, the solution (27) can be recast in the form

$$
\tilde{u}_{n}(k)=\tilde{u}_{n}(\Lambda)\left(\frac{k}{\Lambda}\right)^{-d}\left(\frac{k}{\Lambda}\right)^{\alpha_{d} \tilde{\beta}^{2}(\Lambda) n^{2}+\mathcal{O}\left(\epsilon^{2}\right)} .
$$

One may be tempted to conclude that the couplings $\tilde{u}_{n}(k)$ are relevant, marginal, and irrelevant depending on $\tilde{\beta}^{2}(\Lambda) n^{2}-\frac{d}{\alpha_{d}}<0,=0$, and $>0$, respectively. This distinction is, however, made according to the bare value of the coupling $\tilde{\beta}^{2}(\Lambda)$. The latter goes to infinity in the UV regime (pushing the UV cut-off $\Lambda$ to infinity), so that in fact all couplings $\tilde{u}_{n}(k)$ are irrelevant in the UV regime. As the scale $k$ is decreased, a progressively larger number of couplings with increasing index $n$ become relevant. For example, as Fig. 1 (a) illustrates, the coupling, $\tilde{u}_{1}(k)$ which is irrelevant in the UV regime, becomes relevant along the RG trajectories. In the cross-over region the UV scaling laws lose their validity due to the non-linear terms of higher order. Including the latter and following the RG trajectories through the critical scale $k_{\mathrm{c}}$ down to $k \rightarrow 0$, one does not find any qualitative change in the behaviour of the RG trajectories. This is illustrated in Fig प(b) using the example of a single RG trajectory (see Sect. IIIE for the details of the calculation).

The UV scaling behaviour found for $d \geq 3$ is completely different from that obtained for $d=2$ in Ref. [12] [see Eq. (17) $i b i d$.$] . For d=2$, a strong and a weak coupling phases with different scaling laws have been detected in the UV regime depending on the actual value of the scale-independent parameter $\beta$. For $d \geq 3$ the same UV scaling laws have been found for arbitrary values of $\beta$. Neither the UV scaling laws, nor the full RG-flow diagram indicate the existence of different phases in the lowest order of the gradient expansion. The disappearence of the topological phase transition for $d \geq 3$ of dimensions is of course related to the fact that long-range order is no longer prohibited by the Mermin-Wagner theorem in higher dimensions, and to the interplay of the long-range order with the topological excitations.

\section{E. Effective potential}

We now determine numerically the effective potential $V_{\text {eff }}(\phi)$ for the 4-dimensional GSGM as the limit $k \rightarrow 0$ of the blocked potential $V_{k}(\phi)$. For this purpose the flow equations (21) and (23) have to be solved numerically for $k>k_{c}$ above the scale $k_{c}$ of the spinodal instability, and the minimization steps according to Eq. 25) have to be carried out for $k<k_{c}$ in the region of the spinodal instability. The Fourier-expansion of the periodic potential has to be truncated at the term with $n=N, N$ fixed. Monitoring numerically the coupling constants $\tilde{u}_{n}(k)(1 \leq n \leq N)$ of the periodic field theory, we compare the solution with the results obtained by solving the WH-RG equation (15D) in LPA for the appropriately chosen polynomial potential

$$
V_{k}(\phi)=\sum_{m=1}^{N} \frac{1}{(2 m) !} g_{2 m}(k) \phi^{2 m} .
$$

The polynomial potential was "matched" to the periodic one at the UV scale by equating the bare couplings $g_{2 m}(\Lambda)$ to the corresponding coefficients in the Taylor expansion of the bare periodic potential at $\phi=0$ :

$$
V_{\Lambda}(\phi)=\sum_{n=1}^{N} u_{n}(\Lambda) \cos (n \beta \phi)=\sum_{m=1}^{N} \frac{1}{(2 m) !}\left[(-1)^{m} \beta^{2 m} \sum_{n=1}^{N} u_{n}(\Lambda) n^{2 m}\right] \phi^{2 m} \equiv \sum_{m=1}^{N} \frac{1}{(2 m) !} g_{2 m}(\Lambda) \phi^{2 m} .
$$

In order to avoid the spinodal instability for the polynomial case, the initial conditions for the polynomial potential were chosen specifically as $g_{2}(k=\Lambda)=0.0001, g_{4}(k=\Lambda)=0.1$ and $g_{m}(k=\Lambda)=0$, if $m>4$. In this manner 
for any given values of $\beta^{2}$, and $N$ we get different sets of initial values $\tilde{u}_{n}(\Lambda)$ for the Fourier-amplitudes $\tilde{u}_{n}(k)$. For the comparison to the corresponding polynomial potential, the blocked periodic potential is again decomposed into a Taylor-series at each scale $k$. In this way, as a side-effect, one can investigate the influence of the violation of periodicity due to the Taylor-expansion on the RG flow of the periodic potential.

Equations (21) and (23) are solved numerically, starting from the UV cut-off $\Lambda=1$ by using a fourth-order RungeKutta method with the step size $\delta k=10^{-p} k$ with $p=4$. There were no appreciable changes in the numerical results by increasing $p$ further. The numerics have been tested by reproducing the results of [39] for the polynomial case. Below the critical scale $k_{\mathrm{c}}$ where the spinodal instability occurs for the periodic case, the RG flow was determined by the help of the tree-level blocking relation (25). As mentioned above, this equation has the same form for any number $d$ of dimensions. Therefore, the numerical algorithm used and the function $\rho_{k}(\phi)$ providing the minimum are the same as those for $d=2$ in our previous work [12]. Generally, for $N \geq \mathcal{O}(10)$ Fourier modes a good convergence has been observed at all scales. For $d=4$ the RG flow projected onto the plane $(J, y)$ is qualitatively the same as that for $d=3$ in Fig. 1. This flow diagram shows that no different phases occur in our approximation, but the UV-irrelevant, dimensionless parameter $y$ becomes relevant in the IR regime.

The upper plots in Figs. 2 and 3 and those in Figs. 4 and 5 show the scaling of the dimensionful coupling constants $g_{2}(k)$ and $g_{4}(k)$ above the scale of the spinodal instability for various truncations $N$ of the Fourier expansion of the potential and for the bare values $\tilde{\beta}^{2}(\Lambda)=2 \tilde{\beta}_{0}^{2}=128 \pi^{2}$ and $\tilde{\beta}^{2}(\Lambda)=\frac{1}{2} \tilde{\beta}_{0}^{2}=32 \pi^{2}$, respectively. [According to Eq. (29), $\tilde{u}_{1}(k)$ is independent of the scale $k$ for $\tilde{\beta}^{2}(\Lambda)=\tilde{\beta}_{0}^{2}=64 \pi^{2}$.] We see the good convergence of the numerical results with increasing number $N$ of the included Fourier-modes. There is no significant difference in the extreme UV regime between the solutions for the periodic and the corresponding polynomial cases. Below this narrow UV "window", the scaling laws for the coupling constants of the periodic potential deviate remarkably from those for the corresponding polynomial potential. Namely, the coupling constants for the periodic case start to decrease in magnitude after passing through a cross-over region.

The lower plots in Figs. 2- 5 show the scaling of the coupling constants $g_{2}(k)$ and $g_{4}(k)$ in the region of the spinodal instability for various values of the bare coupling $\tilde{\beta}^{2}(\Lambda)$. The corresponding curves in the lower plots have not been artificially smoothened out in order to demonstrate the relatively small inaccuracy of the numerical calculation. In this deep IR regime the dimensionful coupling constants tend to zero for the limit $k \rightarrow 0$ in an oscillatory manner. Increasing the number $N$ of the encountered Fourier-modes of the blocked potential does not alter this general behaviour. This behaviour is qualitatively different from that for the polynomial case when all dimensionful coupling constants (with even indices) tend to a non-vanishing constant for $k \rightarrow 0$ [26, 39].

As illustrated in Fig. [6] the blocked periodic potential flattens out to a constant in the limit $k \rightarrow 0$. This behaviour is in accordance with the global features of the RG flow discussed in Sect. IIIB In this case the information on the IR physics of the system is encoded in the manner in which the blocked potential tends to a constant value. Because the spinodal instability always occurs, the minima of the blocked potential are filled due to the large-amplitude fluctuations of the field.

\section{RG FLOW OF CG}

\section{A. RS-RG for CG}

In this section we apply the RS-RG method in the dilute gas approximation (DGA) to the renormalization of the $d$-dimensional ECG. The same approach was applied to the two-dimensional generalized Coulomb gas many years ago in [7]. Here, we generalize it for the the partition function (51) with in $d \geq 2$ dimensions while following as close as possible the derivation in [7] for $d=2$. The details of the renormalization procedure are described in App. ¿ The RS-RG in DGA provides the following flow equations for the parameters of the $d$-dimensional ECG:

$$
\begin{aligned}
a \frac{d \tilde{\beta}^{2}}{d a}= & -(d-2) \tilde{\beta}^{2}+\tilde{\beta}^{4} \frac{\Omega_{d}^{2}}{d}\left\{\begin{array}{c}
-c_{2} \\
\frac{1}{2} c_{d}
\end{array}\right\}<\sigma^{2}>, \\
a \frac{d w_{\sigma}}{d a}= & w_{\sigma} d-\frac{1}{2} c_{2} \delta_{d, 2} \tilde{\beta}^{2} w_{\sigma} \sigma^{2}+\frac{1}{2} \Omega_{d} \sum_{\sigma^{\prime} \neq \sigma} w_{\sigma^{\prime}} w_{\sigma-\sigma^{\prime}} \exp \left[-\frac{1}{2} \tilde{\beta}^{2} \sigma^{\prime}\left(\sigma-\sigma^{\prime}\right) \tilde{G}(a)\right] \\
& -w_{\sigma} \frac{\Omega_{d}^{2}}{d} \sum_{\sigma^{\prime} \neq 0} w_{\sigma^{\prime}} w_{-\sigma^{\prime}} \exp \left[\frac{1}{2} \tilde{\beta}^{2} \sigma^{\prime 2} \tilde{G}(a)\right]-\frac{d-2}{4 d} \tilde{\beta}^{4} w_{\sigma} \Omega_{d}^{2} \sigma^{2} \tilde{G}^{2}(a)<{\sigma^{\prime}}^{2}>,
\end{aligned}
$$


where

$$
<\sigma^{2}>=\sum_{\sigma \neq 0} \sigma^{2} w_{\sigma} w_{-\sigma} \exp \left[\frac{1}{2} \tilde{\beta}^{2} \sigma^{2} \tilde{G}(a)\right]
$$

and $\tilde{G}(a)=\left(1-\delta_{d, 2}\right)\left[(d-2) \Omega_{d}\right]^{-1}$. Note that the first and second rows of the curly bracket on the r.h.s. of the first of Eqs. (32) correspond to the cases $d=2$ and $d>2$, respectively.

The well-known flow equations for $d=2$ [7] are recovered by these general formulae. For $d=2$ and $Z(2)$ symmetry, $w_{-\sigma}=w_{\sigma}$, one gets $\tilde{G}(a)=0$ and $\left\langle\sigma^{2}>=2 \sum_{\sigma=1}^{\infty} \sigma^{2} w_{\sigma}^{2}\right.$, and the RG equations take the form:

$$
a \frac{d \tilde{\beta}^{2}}{d a}=-2 \pi \tilde{\beta}^{4} \sum_{\sigma=1}^{\infty} \sigma^{2} w_{\sigma}^{2}, \quad a \frac{d w_{\sigma}}{d a}=\left(2-\frac{\tilde{\beta}^{2}}{4 \pi} \sigma^{2}\right) w_{\sigma}+\pi \sum_{\sigma^{\prime} \neq 0, \sigma} w_{\sigma^{\prime}} w_{\sigma-\sigma^{\prime}}-4 \pi^{2} w_{\sigma} \sum_{\sigma^{\prime}=1}^{\infty} w_{\sigma^{\prime}}^{2} .
$$

Up to numerical prefactors, these equations are the same as Eq. (8) in Sect. IIB which represent the well-known RG equations for the two-dimensional electric Coulomb gas [7]. The extra factor 2 on the r.h.s. of the first equation appears due to the contributions of both charges $\sigma= \pm 1$ to the value of $\left\langle\sigma^{2}\right\rangle$. The other, slightly different factors depend on the different inaccurate determinations of the overlapping regions of integrations for momenta, when the contributions of the annihilation and fusion of charges are calculated (e.g. see the note after Eq. A14).

\section{COMPARISON OF RG FLOW FOR RELATED MODELS}

\section{A. RG flow for GSGM and ECG}

The flow equations for the $d$-dimensional ECG can be compared to those for the GSGM. Such a comparison is meaningful only for the UV regime due to different renormalization schemes used in the two cases. Furthermore, the RS-RG method has the drawback that is not adequate to detect the spinodal instability. This is because the RS-RG applies a sharp cut-off in real space which corresponds to a smooth cut-off in momentum space. Therefore, there is no way to treat modes above and below the critical cut-off $k_{c}$ differently and to detect the spinodal instability in the RS-RG approach.

In order to compare the RG flow of the ECG with that of the GSGM, we rewrite Eqs. (32) in terms of the dimensionless fugacities $\tilde{u}_{\sigma}=a^{d} u_{\sigma}$ by making use of Eq. (6). We also introduce the momentum scale $k \propto a^{-1}$. Then, the flow equations obtained by the RS-RG for the ECG take the form:

$$
\begin{aligned}
k \frac{d \tilde{\beta}^{2}}{d k}= & +(d-2) \tilde{\beta}^{2}-\left(1-3 \delta_{d, 2}\right) \frac{\Omega_{d} \tilde{\beta}^{4}}{4 d} \sum_{\sigma=1}^{\infty} \sigma^{2} \tilde{u}_{\sigma} \tilde{u}_{-\sigma} \exp \left[-\left(1-\delta_{d, 2}\right) \frac{\tilde{\beta}^{2} \sigma^{2}}{2(d-2) \Omega_{d}}\right], \\
k \frac{d \tilde{u}_{\sigma}}{d k}= & -\left(d-\frac{\tilde{\beta}^{2} \sigma^{2}}{2 \Omega_{d}}\right) \tilde{u}_{\sigma}+\frac{\Omega_{d}}{4} \sum_{\sigma^{\prime} \neq 0, \sigma} \tilde{u}_{\sigma^{\prime}} \tilde{u}_{\sigma-\sigma^{\prime}} \exp \left[-\left(1-\delta_{d, 2}\right) \frac{\tilde{\beta}^{2} \sigma^{\prime}\left(\sigma^{\prime}-\sigma\right)}{2(d-2) \Omega_{d}}\right] \\
& +\frac{\Omega_{d}^{2}}{4 d} \tilde{u}_{\sigma} \sum_{\sigma^{\prime} \neq 0} \tilde{u}_{\sigma^{\prime}} \tilde{u}_{-\sigma^{\prime}} \exp \left[-\left(1-\delta_{d, 2}\right) \frac{\tilde{\beta}^{2} \sigma^{\prime 2}}{2(d-2) \Omega_{d}}\right] .
\end{aligned}
$$

These equations should be compared with Eqs. (21) and (24) for the GSGM. As to the tree-level scaling, i.e. the linearized flow equations, the WH-RG LPA and RS-RG DGA give identical results. The non-linear term of the order $\mathcal{O}(\tilde{u})$ on the r.h.s. of Eq. (24) is identical with the corresponding term on the r.h.s. of Eq. (36) for $d=2$ and has a slightly different numerical prefactor for $d \geq 3$. The two methods give completely different results concerning the non-linear terms of the order higher than $\mathcal{O}(\tilde{u})$. This happens because the gradient expansion and the dilute gas approximation cannot be related directly. For comparison, the RG flow may be calculated for both models in $d=3$, and the RG trajectories may be projected onto the plane $\left(J=1 / \tilde{\beta}^{2}, y\right)$ where $y=\tilde{u}_{1}^{2}$ for the GSGM [Fig. [1(b)] and $y=(2 \pi)^{-2} \tilde{u}_{1}^{2} \exp \left[-\tilde{\beta}^{2} /(8 \pi)\right]$ for the ECG [Fig. 目. In the latter case the projected RG trajectories are given by

$$
\begin{aligned}
& a \frac{d J}{d a}=J-\frac{4 \pi^{3}}{3} y, \\
& a \frac{d y}{d a}=\left(6-\frac{1}{8 \pi J}\right) y-\frac{32 \pi^{4}}{3} y^{2}-\frac{\pi^{2}}{6 J^{2}} y^{2} .
\end{aligned}
$$


A significant difference arises due to neglecting the wave-function renormalization in the WH-RG approach for the GSGM. Namely, Eq. (21) valid for the scale-dependence of the 'temperature' at any scale $k$, does not contain any loop-corrections as compared to the corresponding Eq. (35) for the ECG. Therefore, Eq. (21) does not have any fixed point at finite values of $J$ and $y$ (i.e. those of $\tilde{\beta}^{2}$ and $\tilde{u}_{1}$ ) in the flow diagram for the GSGM [Fig. 1(b)]. There exists a UV fixed point $(J \rightarrow 0, y \rightarrow \infty)$ and a zero-temperature fixed point $(J \rightarrow \infty, y \rightarrow \infty)$. The phase diagram for the ECG (Fig. 17) however reveals the existence of a repulsive UV fixed point at finite values of $J$ and $y$, when the terms of the order $\mathcal{O}\left(y^{2}\right)$ are neglected in Eqs. (37b). For small values of the fugacities the non-linear terms are negligible and the RG flow looks rather similarly for both models. The fixed point in Fig. 7 maybe artificial because on the one hand, the RS-RG method in the dilute gas approximation is not well suited to determine the RG flow for large values of the fugacity. On the other hand, the WH-RG in the LPA, the validity of which is not restricted to small fugacity values, does not exhibit any fixed point. Nevertheless the clarification of the role of the wafefunction renormalization in the WH-RG method is an open question and it seems not to be possible in the framework of the gradient expansion.

The comparison of the flow diagram in Fig. 7 with that in Fig. 8 where the terms of the order $\mathcal{O}\left(y^{2}\right)$ are also included, reveals the role of the last two nonlinear terms on the r.h.s. of Eq. (37b). Due to these nonlinearities, the fixed point at finite parameter values turns from a repulsive UV fixed point to an attractive IR one. As to the global features of the flow diagram, this indicates that one certainly has to go beyond the DGA in order to clarify the essential role of the nonlinearities in the RS renormalization of ECG.

\section{B. RG flow for ECG and VLG}

The RG-flow diagram for the ECG presented above may be significantly modified due to non-linear terms of higher order when going beyond the DGA. Nevertheless, it can be compared in the UV regime to the RG-flow diagram for the VLG that was also obtained after certain linearizations 20]. Such a comparison could in principle give more insight to the question posed in the literature (see [10] and the references therein) on whether the VLG and the three-dimensional XY-model (or the three-dimensional Coulomb gas) really belong to the same class of universality, or not. Our strategy is simply to show that there exists a transformation of the parameters $\left(\tilde{\beta}^{2}, \tilde{u}_{1}^{2}\right) \mapsto(K, y)$ which transforms the set of Eqs. (35) and (36) with the charges $\sigma= \pm 1$ for the three-dimensional ECG to the equations of the form very close to that of the set of Eqs. (13) for the VLG up to the non-linear terms of higher order. In fact, introducing the parameters

$$
K=\left(\frac{k_{0}}{k}\right)^{2} \tilde{\beta}^{2}=\left(\frac{a}{a_{0}}\right)^{2} \tilde{\beta}^{2}, \quad y=-\frac{1}{4 \pi^{2}} \tilde{u}_{1}^{2} \exp \left[-\frac{\tilde{\beta}^{2}}{8 \pi}\right],
$$

the flow equations (35), (36) give

$$
a \frac{d K}{d a}=K-\frac{4 \pi^{3}}{3} K^{2} y\left(\frac{a_{0}}{a}\right)^{2}, \quad a \frac{d y}{d a}=\left(6-\frac{K}{8 \pi}\left(\frac{a_{0}}{a}\right)^{2}\right) y+\mathcal{O}\left(y^{2}\right) .
$$

These equations are very similar to Eq. (13), although there is an additional scale dependence in the equation for the flow of the parameter $K$. More questionable is the interpretation of $y$ as fugacity of anything because it is a negative quantity. These discrepancies may reflect the dependence on the different approximations used when the RS-RG has been applied to ECG and VLG, respectively. Therefore, any conclusion on whether the VLG and the three-dimensional Coulomb gas belong to the same universality class, has to be seen as premature at this stage.

\section{SUMMARY}

A renormalization-group analysis of the generalized sine-Gordon model (GSGM) and that of the equivalent Coulomb gas (ECG) have been pursued for $d \geq 3$ dimensions. The RG equations for the GSGM and the ECG are derived in two ways: (i) by means of Wegner's and Houghton's (WH-RG) method in the local-potential approximation and (ii) by the real-space RG (RS-RG) in the dilute-gas approximation. These different approaches complement one another: the WH-RG applied to the GSGM is better suited to determine the IR behaviour of the system, whereas the RS-RG applied to the ECG is able to better represent the non-linearities which influence the UV scaling. It has been shown that the leading-order terms of the flow equations obtained by these methods agree well. Taking as an example the 4-dimensional GSGM, we have demonstrated numerically that the blocked potential tends to a constant effective potential, independent of the field-variable, in the IR limit. This behaviour is in agreement with 
the global requirements on the RG flow that constrain the effective potential to be periodic and convex at the same time. Comparing the evolution of the periodic blocked potential to that of a polynomial potential obtained by a Taylor-expansion of the bare periodic potential, we show that a violation of the periodicity would lead to a completely incorrect IR scaling. The flow diagram in Fig. 1 1 shows that only a single phase occurs in our approximation, and that the UV-irrelevant, dimensionless parameter $y$ becomes relevant in the IR regime.

Because the GSGM, the ECG and the XY-model belong to the same universality class, the determination of the RG flow of these models for the three-dimensional case via two different, complementary methods in the UV regime enables us to compare the UV scaling with that of a three-dimensional vortex gas (VLG) 20]. It has been established that one may transform the flow equations for the ECG to a form very close to that of the flow equations for the VLG, if only the leading order terms are kept. Nevertheless, the negative fugacity one needs to introduce and the different explicit scale-dependences occurring in the two sets of the flow equations do not enable one to make any definitive conclusion regarding the question whether the VLG and the three-dimensional Coulomb gas belong to the same universality class.

\section{Acknowledgments}

This work has been supported by the BMBF, by GSI (Darmstadt) and by the projects OTKA T032501/00, NATO SA(PST.CLG 975722)5066, and MÖB-DAAD 27 (323-PPP-Ungarn). I. N. is also grateful for support by DAAD. Part of the computations have been performed in the Supercomputer Lab. of the Faculty of Natural Sciences of the Univ. of Debrecen grown out from the donation of the Alexander von Humboldt Foundation. I.N. and K.S. are indebted to Zs. Gulácsi and J. Polonyi for extremely useful discussions. One of the authors (I.N.) would like to thank K. Vad for the helpful discussions on the experimental background. 
[1] J. M. Kosterlitz, D. J. Thouless, J. Phys. C6 (1973) 118; J. M. Kosterlitz, J. Phys. C7 (1974) 1046.

[2] S. Coleman, Phy. Rev. D11 (1975) 3424.

[3] J. V. Jose, L. P. Kadanoff, S. Kirkpatrick, D. R. Nelson, Phys. Rev. B16 (1977) 1217.

[4] P. B. Wiegmann, J. Phys. C11 (1978) 1583.

[5] S. Samuel, Phys. Rev. D18 (1978) 1916.

[6] D. Amit, Y. Y. Goldschmidt, G. Grinstein, J. Phys. A13 (1980) 585.

[7] B. Nienhuis in Phase Transitions and Critical Phenomena, Vol. 11 ed. by C. Domb, J.L. Lebowitz (Academic Press, London, 1987), 1-53.

[8] K. Huang, J. Polonyi, Int. J. Mod. Phys. A6 (1991) 409.

[9] R. J. Creswick, H. A. Farach, C. P. Poole, Jr. Introduction to RG methods in Physics, (Wiley, New York, 1992)

[10] Zs. Gulácsi, M. Gulácsi, Adv. in Phys. 47 (1998) 1-89.

[11] G. v. Gersdorff, C. Wetterich, Phys. Rev. B64 (2001) 054513.

[12] I. Nándori, J. Polonyi, K. Sailer, Phys. Rev. D 63 (2001) 045022.

[13] I. Nándori, J. Polonyi, K. Sailer, Phil. Mag. B 81 (2001) 1615.

[14] I. Nándori, K. Sailer, U. D. Jentschura, G. Soff, J. Phys. G 28 (2002) 607.

[15] H. A. Fertig, Kingshuk Majumdar, cond-mat/0302012

[16] J. Polonyi, in Quark Gluon Plasma, World Scientific, 1990, R. Hwa, ed.; K. Johnson, L. Lellouch, J. Polonyi, Nucl. Phys. B367 (1991) 675; Act. Phys. Hung. 2 (1995) 123.

[17] R. Savit, Rev. Mod. Phys. 52, (1980) 453.

[18] F. J. Wegner, A. Houghton, Phys. Rev. A8 (1973) 401.

[19] D. Nelson and D. Fisher, Phys. Rev. B16 (1977) 4945.

[20] S. R. Shenoy, Phys. Rev. B40 (1989) 5056.

[21] M. Campostrini, M. Hasenbusch, A. Pelisetto, P. Rossi, E. Vicari, Phys. Rev. B63 (2001) 214503

[22] M. S. Choi, S. I. Lee, Phys. Rev. B51 (1995) 6680.

[23] S. R. Shenoy, B. Chattapadhyay, Phys. Rev. B51 (1995) 9129.

[24] B. Sas et al., Phys. Rev. B61 (2000) 9118; I. Pethes et al., Synthetic Metals 120 (2001) 1013; F. Portier et al., Phys. Rev. B66 (2002) 140511; K. Vad et al., 2003, to be published.

[25] R. J. Rivers, Path Integral Methods in Quantum Field Theory, (University Press, Cambridge, 1987).

[26] J. Alexandre, J. Polonyi, Phys. Lett. B 445 (1999) 351.

[27] T.R. Morris, J.F. Tighe, JHEP 9908 (1999) 007, T.R. Morris, M.D. Turner, Nucl. Phys. B509 (1998) 637, T.R. Morris, Nucl. Phys. B495 (1997) 477, ibid. Int. J. Mod. Phys. B12 (1998) 1343.

[28] N.D. Mermin, H. Wagner, Phys. Rev. Lett. 17 (1966) 1133.

[29] K. G. Wilson, J. Kogut, Phys. Rep. C12 (1974) 77; K. G. Wilson, Rev. Mod. Phys. 47 (1975) 773; Rev. Mod. Phys. 55 (1983) 583.

[30] J. Polchinski, Nucl. Phys. B231 (1984) 269.

[31] J. Comellas, Nucl. Phys. B509 (1998) 662

[32] R. D. Ball, P. E. Haagensen, J. I. Latorre, E. Moreno, Phys. Lett. B347 (1995) 80

[33] J. Polonyi, K. Sailer, cond-mat/0108179 Phys. Rev. B66 (2002) 155113.

[34] J. Comellas, A. Travesset, Nucl. Phys. B 498 (1997) 2411.

[35] S. Liao, J. Polonyi, Ann. Phys. 222 (1993) 122.

[36] A. Hasenfratz, P. Hasenfratz, Nucl. Phys. B270 (1986) 687.

[37] J. Polonyi, Lectures on Functional Renormalization Group Method, hep-th/0110026

[38] C. Bagnuis, C. Bervillier, Phys. Rept. 348 (2001) 91

[39] J. M. Carmona, J. Polonyi, A. Tarancón, Phys. Rev. D61 (2000) 085018 


\section{APPENDIX A: RS-RG FOR THE CG}

Here, we perform a real-space renormalization in the dilute gas approximation (DGA) of the CG given by the partition function (5). In order to follow as close as possible the procedure employed for $d=2$ (see [10] and references therein), we use continuum integrals over the infinitely large volume $D_{0}$, but cut out spheres $K_{j}$ of radii $a$ at each Coulomb charge $\sigma_{j}$ centered at $x_{j}$ (this is different from lattice regularization). Thus, the regions of integration for the positions of the charges are $D_{0}, D_{1}=D_{0}-K_{1}, D_{2}=D_{0}-K_{1}-K_{2}, \ldots, D_{\nu-1}=D_{0}-K_{1}-\ldots-K_{\nu-1}$ for the charges $\sigma_{1}, \sigma_{2}, \ldots, \sigma_{\nu}$, respectively.

The RG flow equations can be found by changing the resolution, i.e. the smallest separation distance from $a$ to $a+\delta a$ at which two charges can still be detected separately. This means that if two charges become closer than the separation distance $a+\delta a$ they will be seen as a single charge identical to the sum of the two original ones. The partition function will evolve due to the following reasons:

1. the rescaling of the length,

2. the fusion of charges in a single, non-vanishing one,

3. the annihilation, i.e. fusion of charges in a single, neutral one.

These sub-processes acting on the configurations with the number $\nu$ of charges (described by $Z^{[\nu]}$ ) give contributions $\delta_{r s} Z^{[\nu]}, \delta_{f} Z^{[\nu-1]}$, and $\delta_{a} Z^{[\nu-2]}$ to the configurations with the numbers $\nu$-, $(\nu-1)$-, and $(\nu-2)$ of charges, respectively, i.e.

$$
\delta Z=\sum_{\nu=0}^{\infty} \delta_{r s} Z^{[\nu]}+\sum_{\nu=1}^{\infty} \delta_{f} Z^{[\nu-1]}+\sum_{\nu=2}^{\infty} \delta_{a} Z^{[\nu-2]} .
$$

The RG idea is realized by recasting the modified partition sum $Z+\delta Z$ in the original form observed by $Z$, but with modified parameters $\tilde{\beta}^{2}$ and $w_{\sigma}$. Below we determine all these contributions.

1. Rescaling. Rescaling means the change of all integration variables $x_{j}$ to $x_{j}^{\prime}=x_{j}(1+\delta a / a)$ and increasing the size of all regions $D_{j}, K_{j}$ correspondingly. Then we rewrite the partition sum as an integral over the original variables and regions. First of all, the rescaling the volume elements $d^{d} x$ increases them by $(\delta a / a) d d^{d} x$; this can be merged into a modification of the fugacities,

$$
\delta_{r s, 1} w_{\sigma}=w_{\sigma} d \frac{\delta a}{a}
$$

The change of the propagator should be treated differently for $d=2$ and $d \geq 3$.

The case $d=2$. The propagator changes according to

$$
\delta_{r s} \tilde{G}(x)=-c_{2} \frac{\delta a}{a}
$$

(independent of $x$ ). In the interaction energy this yields a contribution

$$
-\frac{1}{2} \tilde{\beta}^{2} \sum_{j, i \neq j} \sigma_{i} \sigma_{j}\left(-c_{2}\right) \frac{\delta a}{a} .
$$

However, for neutral configurations we have $\sum_{j=1}^{\nu} \sigma_{j}=0$, i.e. $\sum_{i \neq j} \sigma_{i}=-\sigma_{j}$, so that one gets the energy contribution

$$
-\frac{1}{2} c_{2} \tilde{\beta}^{2} \sum_{i=1}^{\nu} \sigma_{i}^{2} \frac{\delta a}{a}
$$

which leads to the following modification of the fugacities:

$$
\delta_{r s, 2} w_{\sigma}=w_{\sigma}\left(\exp \left[-\frac{1}{2} c_{2} \tilde{\beta}^{2} \sigma^{2} \frac{\delta a}{a}\right]-1\right)=-\frac{1}{2} c_{2} \tilde{\beta}^{2} w_{\sigma} \sigma^{2} \frac{\delta a}{a} .
$$

The case $d \geq 3$. The change of the propagator,

$$
\delta_{r s} \tilde{G}(x)=-(d-2) \tilde{G}(x) \frac{\delta a}{a},
$$


is proportional to the propagator itself. Therefore, it can be encoded in the change of the coupling $\tilde{\beta}^{2}$ :

$$
\delta_{r s} \tilde{\beta}^{2}=-(d-2) \tilde{\beta}^{2} \frac{\delta a}{a} .
$$

Thus, we get for any numbers $d \geq 2$ of dimensions

$$
\delta_{r s} \tilde{\beta}^{2}=-(d-2) \tilde{\beta}^{2} \frac{\delta a}{a}, \quad \delta_{r s} w_{\sigma}=w_{\sigma} d \frac{\delta a}{a}-\frac{1}{2} c_{2} \delta_{d, 2} \tilde{\beta}^{2} w_{\sigma} \sigma^{2} \frac{\delta a}{a} .
$$

\section{Fusion.}

Let us denote the sphere of radius $a+\delta a$ centered at $x_{j}$ by $K_{j}^{\prime}$ and the annulus of inner radius $a$ and thickness $\delta a$ centered at $x_{j}$ by $\delta_{j}$. Then the regions of intergration in the rescaled partition function are $D_{0}, D_{1}=$ $D_{0}-K_{1}^{\prime}+\delta_{1}=D_{1}^{\prime}+\delta_{1}, D_{2}=D_{0}-K_{1}^{\prime}-K_{2}^{\prime}+\delta_{1}+\delta_{2}$, etc. for $x_{1}, x_{2}$, etc. respectively. The contributions of both the fusion and annihilation of the point charges $\sigma_{p}$ and $\sigma_{q}$ are included in the expression

$$
\begin{aligned}
\delta_{f, a} Z= & \sum_{\nu=0}^{\infty} \frac{1}{\nu !}\left[\sum_{p=2}^{\nu} \sum_{q=1}^{p-1} \int_{D_{0}} \frac{d^{d} x_{1}}{a^{d}} \cdots \int_{D_{q-2}^{\prime}} \frac{d^{d} x_{q-1}}{a^{d}} \int_{D_{q-1}^{\prime}} \frac{d^{d} x_{q}}{a^{d}} \int_{D_{q}^{\prime}} \frac{d^{d} x_{q+1}}{a^{d}} \cdots \int_{D_{p-2}^{\prime}} \frac{d^{d} x_{p-1}}{a^{d}} \int_{\delta_{p}} \frac{d^{d} x_{p}}{a^{d}}\right. \\
& \left.\int_{D_{p}^{\prime}} \frac{d^{d} x_{p+1}}{a^{d}} \cdots \int_{D_{\nu-1}^{\prime}} \frac{d^{d} x_{\nu}}{a^{d}}+\mathcal{O}\left(\frac{\delta a}{a}\right)^{2}\right] \sum_{\sigma_{1}, \ldots, \sigma_{\nu} \neq 0} w_{\sigma_{1}} \cdots w_{\sigma_{\nu}} J_{x_{1}, \ldots, x_{\nu}}^{\sigma_{1}, \ldots, \sigma_{\nu}} .
\end{aligned}
$$

Hence, we have to calculate integrals of the form

$$
I=\int_{D_{0}-K_{z}} \frac{d^{d} x}{a^{d}} \int_{\delta_{x}} \frac{d^{d} y}{a^{d}} f(x, y) \equiv I_{1}+I_{2},
$$

where the point $z$ satisfies either $|x-z|>2 a$ for $I_{1}$, or $a<|x-z|<2 a$ for $I_{2}$. Therefore, the integral for $y$ in $I_{2}$ extends only over the part $\bar{\delta}_{x}$ of the annulus $\delta_{x}$ lying outside of $K_{z}$ :

$$
I_{1}=\int_{|x-z|>2 a} \frac{d^{d} x}{a^{d}} \int_{\delta_{x}} \frac{d^{d} y}{a^{d}} f(x, y), \quad I_{2}=\int_{a<|x-z|<2 a} \frac{d^{d} x}{a^{d}} \int_{\bar{\delta}_{x}} \frac{d^{d} y}{a^{d}} f(x, y) .
$$

Introducing the center of mass coordinate $t=\frac{1}{2}(x+y)$ and the separation coordinate $s=x-y$, one gets

$$
I_{1} \approx \int_{\frac{3}{2} a<|t|} \frac{d^{d} t}{a^{d}} \Omega_{d} \frac{\delta a}{a} f(t, s=a), \quad I_{2} \approx \int_{a<|t|<\frac{3}{2} a} \frac{d^{d} t}{a^{d}} \frac{1}{2} \Omega_{d} \frac{\delta a}{a} f(t, s=a) .
$$

Then, the integral $I$ can be estimated as

$$
I=I_{1}+I_{2} \approx\left[\int_{\frac{3}{2} a<|t|}+\frac{1}{2} \int_{a<|t|<\frac{3}{2} a}\right] \frac{d^{d} t}{a^{d}} \Omega_{d} \frac{\delta a}{a} f(t, s=a) \approx \int_{D_{0}-K_{z}} \omega_{d} \frac{\delta a}{a} f(t, s=a) .
$$

As to the regions of integration, we make another approximation: in accordance with the relation $K_{t}\left(\frac{1}{2} a\right) \subset$ $K_{q} \cup K_{p} \subset K_{t}\left(\frac{3}{2} a\right)$, (the arguments indicate the radii of the spheres) we replace $K_{q} \cup K_{p}$ by $K_{t}$. Due to these approximations terms which are polynomials in a may be neglected. Therefore, our approximations will cease to work as $a$ increases, so that our results are restricted to the UV scaling region. In order to take all terms of the order $\mathcal{O}\left(\frac{\delta a}{a}\right)$ into account, we perform a Taylor-expansion of the function $f(t, s)$ in the variable $s$ before the integration over the annulus. Thus, we find

$$
\begin{aligned}
\delta_{f, a} Z= & \sum_{\nu=0}^{\infty} \frac{1}{\nu !}\left[\sum_{p=2}^{\nu} \sum_{q=1}^{p-1} \int_{D_{0}} \frac{d^{d} x_{1}}{a^{d}} \cdots \int_{D_{q-2}^{\prime}} \frac{d^{d} x_{q-1}}{a^{d}} \int_{D_{q-1}^{\prime}} \frac{d^{d} t}{a^{d}} \int_{D_{q}^{\prime}} \frac{d^{d} x_{q+1}}{a^{d}}\right. \\
& \left.\ldots \int_{D_{p-2}^{\prime}} \frac{d^{d} x_{p-1}}{a^{d}} \int_{D_{p}^{\prime}} \frac{d^{d} x_{p+1}}{a^{d}} \cdots \int_{D_{\nu-1}^{\prime}} \frac{d^{d} x_{\nu}}{a^{d}}+\mathcal{O}\left(\frac{\delta a}{a}\right)^{2}\right] \\
& \left.\sum_{\sigma_{1}, \ldots, \sigma_{\nu} \neq 0} w_{\sigma_{1}} \cdots w_{\sigma_{\nu}} \int_{a<|s|<a+\delta a} \frac{d^{d} s}{a^{d}}\left(1+s_{\alpha} \partial_{\alpha}^{s}+\frac{1}{2} s_{\alpha} s_{\beta} \partial_{\alpha}^{s} \partial_{\beta}^{s}+\mathcal{O}\left(s^{3}\right)\right) J_{x_{1}, \ldots, x_{\nu}}^{\sigma_{1}, \ldots, \sigma_{\nu}}\right|_{s=a} .
\end{aligned}
$$


In order to evaluate this, we expand the exponent first,

$$
\begin{aligned}
J_{x_{1}, \ldots, x_{\nu}}^{\sigma_{1}, \ldots, \sigma_{\nu}} \equiv J^{[\nu]}= & J^{[\nu-2]} \exp \left\{-\tilde{\beta}^{2} \sum_{j \neq p, q} \sigma_{j} \sigma_{p} \tilde{G}_{x_{j}, t+\frac{1}{2} s}-\tilde{\beta}^{2} \sum_{j \neq p, q} \sigma_{j} \sigma_{q} \tilde{G}_{x_{j}, t-\frac{1}{2} s}-\frac{1}{2} \tilde{\beta}^{2} \sigma_{p} \sigma_{q} \tilde{G}(s)\right\} \\
\approx & J^{[\nu-2]} \exp \left\{-\frac{1}{2} \tilde{\beta}^{2} \sigma_{p} \sigma_{q} \tilde{G}(a)\right\} \exp \left\{-\tilde{\beta}^{2} \sum_{j \neq p, q} \sigma_{j} \sigma_{p}\left[1+\frac{1}{2} s_{\alpha} \partial_{\alpha}^{t}+\frac{1}{8} s_{\alpha} s_{\beta} \partial_{\alpha}^{t} \partial_{\beta}^{t}\right] \tilde{G}_{x_{j}, t}\right. \\
& \left.-\tilde{\beta}^{2} \sum_{j \neq p, q} \sigma_{j} \sigma_{q}\left[1-\frac{1}{2} s_{\alpha} \partial_{\alpha}^{t}+\frac{1}{8} s_{\alpha} s_{\beta} \partial_{\alpha}^{t} \partial_{\beta}^{t}\right] \tilde{G}_{x_{j}, t}\right\} .
\end{aligned}
$$

Expressions (A15) and (A16) represent the starting point for the determination of the contribution of the fusion and annihilation of charges to the partition sum. In case of fusion, when the charges $\sigma_{p}$ and $\sigma_{q}$ fuse into a single, non-vanishing charge $\sigma_{t}=\sigma_{p}+\sigma_{q} \neq 0$, the only contribution of the order $\mathcal{O}(\delta a / a)$ comes from the zeroth power of $s$ in (A16). Renumerating the charges in the manner that the new charge at $t=x_{\nu-1}$ becomes the $(\nu-1)$-th one, we can write

$$
\begin{aligned}
\int_{D_{\nu-2}^{\prime}} \frac{d^{d} t}{a^{d}} \int_{a<|s|<a+\delta a} \frac{d^{d} s}{a^{d}} J^{[\nu]} & \approx J^{[\nu-2]} e^{-\frac{1}{2} \tilde{\beta}^{2} \sigma_{p} \sigma_{q} \tilde{G}(a)} \int_{D_{\nu-2}^{\prime}} \frac{d^{d} t}{a^{d}} e^{-\tilde{\beta}^{2} \sum_{j \neq p, q} \sigma_{j} \sigma_{t} \tilde{G}_{x_{j}, t}} \Omega_{d} \frac{\delta a}{a} \\
& =J^{[\nu-1]} e^{-\frac{1}{2} \tilde{\beta}^{2} \sigma_{p} \sigma_{q} \tilde{G}(a)} \Omega_{d} \frac{\delta a}{a} .
\end{aligned}
$$

The other terms with $\int_{\delta_{p}} s^{m} d^{d} s \sim \mathcal{O}\left(a^{m}\right) \frac{\delta a}{a}$ where $m=1,2, \ldots$ are neglected. Inserting this in the change of the partition function due to fusion,

$$
\begin{aligned}
\sum_{\nu=1}^{\infty} \delta Z^{[\nu-1]}= & \sum_{\nu=1}^{\infty} \frac{1}{\nu !} \frac{1}{2} \nu(\nu-1) \int_{D_{0}} \frac{d^{d} x_{1}}{a^{d}} \cdots \int_{D_{\nu-2}^{\prime}} \frac{d^{d} x_{\nu-1}}{a^{d}} \sum_{\sigma_{1} \neq 0} w_{\sigma_{1}} \cdots \sum_{\sigma_{q-1} \neq 0} w_{\sigma_{q-1}} \sum_{\sigma_{q+1} \neq 0} w_{\sigma_{q+1}} \cdots \\
& \sum_{\sigma_{p-1} \neq 0} w_{\sigma_{p-1}} \sum_{\sigma_{p+1} \neq 0} w_{\sigma_{p+1}} \cdots \sum_{\sigma_{\nu} \neq 0} w_{\sigma_{\nu}} \Omega_{d} \frac{\delta a}{a} \sum_{\sigma_{p} \neq 0} w_{\sigma_{p}} \sum_{\sigma_{q} \neq 0} w_{\sigma_{q}}\left[1-\delta_{\sigma_{p},-\sigma_{q}}\right] J^{[\nu-1]} e^{-\frac{1}{2} \tilde{\beta}^{2} \sigma_{p} \sigma_{q} \tilde{G}(a)} \\
= & \sum_{\nu=1}^{\infty} \frac{1}{(\nu-2) !} \int_{D_{0}} \frac{d^{d} x_{1}}{a^{d}} \cdots \int_{D_{\nu-2}^{\prime}} \frac{d^{d} x_{\nu-1}}{a^{d}} \sum_{\sigma_{1} \neq 0} w_{\sigma_{1}} \cdots \sum_{\sigma_{\nu-2} \neq 0} w_{\sigma_{\nu-2}} \sum_{\sigma_{t}=\sigma_{\nu-1} \neq 0} w_{\sigma_{\nu-1}} J^{[\nu-1]} .(\mathrm{A} 18
\end{aligned}
$$

A calculation of this expression finally yields

$$
\delta_{f} w_{\sigma}=\frac{1}{2} \Omega_{d} \frac{\delta a}{a} \sum_{\sigma^{\prime} \neq \sigma} w_{\sigma^{\prime}} w_{\sigma-\sigma^{\prime}} \exp \left[-\frac{1}{2} \tilde{\beta}^{2} \sigma^{\prime}\left(\sigma-\sigma^{\prime}\right) \tilde{G}(a)\right] .
$$

Thus, fusion generates a renormalization of the fugacities. In the manipulations above, we considered all charge configurations as identical if they can be obtained from a given one via permutations only.

\section{Annihilation.}

The charges $\sigma_{p}$ and $\sigma_{q}=-\sigma_{p}$ fuse into a neutral particle when the inequality $a<\left|x_{p}-x_{q}\right|<a+\delta a$ holds for their distance. This is called annihilation, since both charges vanish from the gas. Effectively, the term $Z^{[\nu]}$ then yields a contribution to $Z^{[\nu-2]}$. In Eqs. A15 and A16 one has to keep the terms quadratic in the separation coordinate $s$ (those of the order $a^{2(d-2)}$ in the exponent of $J^{[\nu]}$ ) in order to find all terms of the order $\mathcal{O}(\delta a / a)$. 
Instead of the integral (A17), we now get

$$
\begin{aligned}
& \int_{D_{\nu-2}^{\prime}} \frac{d^{d} t}{a^{d}} \int_{a<|s|<a+\delta a} \frac{d^{d} s}{a^{d}} J^{[\nu]}=J^{[\nu-2]} \exp \left[+\frac{1}{2} \tilde{\beta}^{2} \sigma_{p}^{2} \tilde{G}(a)\right] \\
& \times \int_{D_{\nu-2}^{\prime}} \frac{d^{d} t}{a^{d}} \int_{a<|s|<a+\delta a} \frac{d^{d} s}{a^{d}} \exp \left[-\tilde{\beta}^{2} \sum_{j \neq p, q} \sigma_{j} \sigma_{p} s_{\alpha} \partial_{\alpha}^{t} \tilde{G}_{x_{j}, t}+\mathcal{O}\left(s^{3}\right)\right] \\
& \approx J^{[\nu-2]} \exp \left[\frac{1}{2} \tilde{\beta}^{2} \sigma_{p}^{2} \tilde{G}(a)\right] \int_{D_{\nu-2}^{\prime}} \frac{d^{d} t}{a^{d}} \int_{a<|s|<a+\delta a} \frac{d^{d} s}{a^{d}} \\
& {\left[1-\tilde{\beta}^{2} \sum_{j \neq p, q} \sigma_{j} \sigma_{p} s_{\alpha} \partial_{\alpha}^{t} \tilde{G}_{x_{j}, t}+\frac{1}{2} \tilde{\beta}^{4} \sigma_{p}^{2} \sum_{j \neq p, q} \sum_{i \neq p, q} \sigma_{j} \sigma_{i} s_{\alpha} s_{\beta} \partial_{\alpha}^{t} \tilde{G}_{x_{j}, t} \partial_{\beta}^{t} \tilde{G}_{x_{i}, t}\right] .}
\end{aligned}
$$

Here the linear term and the off-diagonal part of the matrix $s_{\alpha} s_{\beta}$ give vanishing contributions to the integral over the annulus, so that one gets

$$
\begin{aligned}
\int_{D_{\nu-2}^{\prime}} \frac{d^{d} t}{a^{d}} \int_{a<|s|<a+\delta a} \frac{d^{d} s}{a^{d}} J^{[\nu]} \approx & J^{[\nu-2]} \exp \left[\frac{1}{2} \tilde{\beta}^{2} \sigma_{p}^{2} \tilde{G}(a)\right] \int_{D_{\nu-2}^{\prime}} \frac{d^{d} t}{a^{d}} \int_{a<|s|<a+\delta a} \frac{d^{d} s}{a^{d}} \\
& \times\left[1+\frac{1}{2 d} s^{2} \tilde{\beta}^{4} \sigma_{p}^{2} \sum_{j \neq p, q} \sum_{i \neq p, q} \sigma_{j} \sigma_{i} \partial_{\alpha}^{t} \tilde{G}_{x_{j}, t} \partial_{\alpha}^{t} \tilde{G}_{x_{i}, t}\right] .
\end{aligned}
$$

We now change the order of integration so that the annihilating charges are the "last ones in the queue" and integrate by parts in the second term, with the result

$$
\begin{aligned}
\int_{D_{\nu-2}^{\prime}} \frac{d^{d} t}{a^{d}} \int_{a<|s|<a+\delta a} \frac{d^{d} s}{a^{d}} J^{[\nu] \approx} & J^{[\nu-2]} \exp \left[\frac{1}{2} \tilde{\beta}^{2} \sigma_{p}^{2} \tilde{G}(a)\right] \Omega_{d} \frac{\delta a}{a} \\
& \left\{\int_{D_{\nu-2}^{\prime}} \frac{d^{d} t}{a^{d}} 1+\frac{1}{2 d} \tilde{\beta}^{4} a^{2-d} \sigma_{p}^{2} \sum_{j, i=1}^{\nu-2} \sigma_{j} \sigma_{i}\right. \\
& \left(\oint_{\partial D_{0}}-\sum_{l=1}^{\nu-2} \oint_{\partial K_{l}}\right) d \sigma_{\alpha} \tilde{G}_{x_{j}, t} \partial_{\alpha}^{t} \tilde{G}_{x_{i}, t} \\
& \left.-\frac{1}{2 d} \tilde{\beta}^{4} a^{2-d} \sigma_{p}^{2} \sum_{j, i=1}^{\nu-2} \int_{D_{\nu-2}^{\prime}} \frac{d^{d} t}{a^{d}} \tilde{G}_{x_{j}, t} \partial^{t} \partial^{t} \tilde{G}_{x_{i}, t}\right\},
\end{aligned}
$$

where the last integral vanishes since $\partial \partial G(x) \sim \delta(x)$ and the point $t=x_{i}$ is excluded from the region of integration. The first term on the r.h.s. can be included in the renormalization of the free energy which, however, we do not consider here. Therefore, the only nonvanishing terms originate from the surface integrals on the r.h.s. of Eq. (A22). They should be considered separately. The integral over the surface of the domain $D_{0}$ vanishes as the integrand goes sufficiently fast to zero for $|t| \rightarrow \infty$. The integrand of the integral over the surface $\partial K_{l}$ of the sphere centered at $x_{l}$ contains the derivative

$$
\partial_{\alpha}^{t} \tilde{G}_{x_{i}, t}=\frac{\left(t-x_{i}\right)_{\alpha}}{\left|t-x_{i}\right|^{2}} \begin{cases}-c_{2}, & \text { for } \quad d=2 \\ \frac{1}{2}(d-2) \tilde{G}_{x_{i}, t}, & \text { for } \quad d \geq 3\end{cases}
$$

Using the outer normal $n_{\alpha}$ of the surface $\partial K_{l}$ and writing $t=x_{l}+a n$, we obtain:

$$
\oint_{\partial K_{l}} d \sigma_{\alpha} \tilde{G}_{x_{j}, t} \partial_{\alpha}^{t} \tilde{G}_{x_{i}, t}=\oint_{\partial K_{l}} d f n_{\alpha} \tilde{G}\left(x_{l}+a n-x_{j}\right) \partial_{\alpha}^{l} \tilde{G}\left(x_{l}+a n-x_{i}\right)=: I .
$$

Here we have to distinguish the following particular cases: 
(a) For $l \neq i, j$ :

$$
\begin{aligned}
I= & \oint_{\partial K_{l}} d f n_{\alpha}\left[\tilde{G}\left(x_{l}-x_{j}\right) \partial_{\alpha}^{l} \tilde{G}\left(x_{l}-x_{i}\right)\right. \\
& \left.+a n_{\beta} \partial_{\beta}^{l} \tilde{G}\left(x_{l}-x_{j}\right) \partial_{\alpha}^{l} \tilde{G}\left(x_{l}-x_{i}\right)+a n_{\beta} \tilde{G}\left(x_{l}-x_{j}\right) \partial_{\beta}^{l} \partial_{\alpha}^{l} \tilde{G}\left(x_{l}-x_{i}\right)\right] .
\end{aligned}
$$

Here, the first term vanishes. The last term vanishes too as it is proportional to $\delta\left(x_{l}-x_{i}\right)$, but we have explicitly $x_{l} \neq x_{i}$. The second term can be evaluated as

$$
\approx \Omega_{d} \frac{a^{d}}{d} \partial_{\alpha}^{l} \tilde{G}\left(x_{l}-x_{j}\right) \partial_{\alpha}^{l} \tilde{G}\left(x_{l}-x_{i}\right)=\Omega_{d} \frac{a^{d}}{d} \frac{\left(x_{l, j}\right)_{\alpha}\left(x_{l, i}\right)_{\alpha}}{\left|x_{l, j}\right|^{2}\left|x_{l, i}\right|^{2}} \begin{cases}c_{2}^{2}, & \text { for } \quad d=2, \\ \frac{1}{4}(d-2)^{2} \tilde{G}\left(x_{l, j}\right) \tilde{G}\left(x_{l, i}\right), & \text { for } \quad d \geq 3,\end{cases}
$$

with $x_{l, j}=x_{l}-x_{j}$.

(b) For $l=j \neq i$ :

$$
\begin{aligned}
I & =\oint_{\partial K_{l}} d \sigma_{\alpha} \tilde{G}_{x_{j}, t} \partial_{\alpha}^{t} \tilde{G}_{x_{i}, t}=\oint_{\partial K_{l}} d f n_{\alpha} \tilde{G}(a n) \partial_{\alpha}^{l} \tilde{G}\left(x_{l}+a n-x_{i}\right) \\
& \approx \oint_{\partial K_{l}} d f n_{\alpha} \tilde{G}(a n)\left[\partial_{\alpha}^{l} \tilde{G}\left(x_{l, i}\right)+a n_{\beta} \partial_{\alpha}^{l} \partial_{\beta}^{l} \tilde{G}\left(x_{l, i}\right)\right]=0,
\end{aligned}
$$

where the first term vanishes due to isotropy and the second one vanishes, too, for the same reason as the last term on the r.h.s. of Eq. A25.

(c) For $l=i \neq j$ :

$$
\begin{aligned}
I & =\oint_{\partial K_{l}} d \sigma_{\alpha} \tilde{G}_{x_{j}, t} \partial_{\alpha}^{t} \tilde{G}_{x_{i}, t} \\
& =\left.\oint_{\partial K_{l}} d f n_{\alpha} \tilde{G}\left(x_{l}+a n-x_{j}\right) \partial_{\alpha}^{y} \tilde{G}(y)\right|_{y=a n} \\
& \left.\approx \oint_{\partial K_{l}} d f n_{\alpha}\left[\tilde{G}\left(x_{l, j}\right)+a n_{\beta} \partial_{\beta}^{l} \tilde{G}\left(x_{l, j}\right)\right] \partial_{\alpha}^{y} \tilde{G}(y)\right|_{y=a n}
\end{aligned}
$$

(d) For $l=i=j$ :

$$
\begin{aligned}
I & =\oint_{\partial K_{l}} d \sigma_{\alpha} \tilde{G}_{x_{j}, t} \partial_{\alpha}^{t} \tilde{G}_{x_{i}, t} \\
& =\left.\oint_{\partial K_{l}} d f n_{\alpha} \tilde{G}(\text { an }) \partial_{\alpha}^{y} \tilde{G}(y)\right|_{y=a n} \\
& = \begin{cases}0, & \text { for } \quad d=2, \\
\frac{1}{2} \Omega_{d} a^{d-2}(d-2) \tilde{G}^{2}(a), & \text { for } \quad d \geq 3 .\end{cases}
\end{aligned}
$$


By employing all the above results, we are now finally in a position to rewrite the r.h.s. of Eq. (A22):

$$
\begin{aligned}
& J^{[\nu-2]} \exp \left[\frac{1}{2} \tilde{\beta}^{2} \sigma_{p}^{2} \tilde{G}(a)\right] \Omega_{d} \frac{\delta a}{a} \frac{1}{a^{d}}\left[T V-(\nu-2) \frac{\Omega_{d} a^{d}}{d}\right. \\
& -\frac{1}{2 d} \tilde{\beta}^{4} a^{2-d} \sigma_{p}^{2}\left[\sum_{j, i=1}^{\nu-2} \sigma_{j} \sigma_{i} \sum_{l \neq i, j}^{\nu-2} \Omega_{d} \frac{a^{d}}{d} \frac{\left(x_{l, j}\right)_{\alpha}\left(x_{l, i}\right)_{\alpha}}{\left|x_{l, j}\right|^{2}\left|x_{l, i}\right|^{2}}\left\{\begin{array}{l}
c_{2}^{2} \\
\frac{1}{4}(d-2)^{2} \tilde{G}\left(x_{l, j}\right) \tilde{G}\left(x_{l, i}\right)
\end{array}\right\}\right. \\
& \left.\left.+\sum_{j}^{\nu-2} \sigma_{j} \sum_{l \neq j}^{\nu-2} \sigma_{l} \Omega_{d} a^{d-2} \tilde{G}\left(x_{l, j}\right)\left\{\begin{array}{l}
c_{2} \\
\frac{1}{2}(d-2) \tilde{G}(a)
\end{array}\right\}+\sum_{j}^{\nu-2} \sigma_{j}^{2} \frac{1}{2} \Omega_{d} a^{d-2}(d-2) \tilde{G}^{2}(a)\right]\right],
\end{aligned}
$$

where, again, the first and second rows of the columns stand for $d=2$ and $d \geq 3$, respectively. Multiplying this expression by $w_{\sigma_{p}} w_{-\sigma_{p}}$ and summing up for $\sigma_{p}$, one obtains:

$$
\begin{aligned}
J^{[\nu-2]} \Omega_{d} & \frac{\delta a}{a}\left[\frac{1}{a^{d}}\left(T V-(\nu-2) \frac{\Omega_{d} a^{d}}{d}\right) \sum_{\sigma \neq 0} w_{\sigma} w_{-\sigma} \exp \left[\frac{1}{2} \tilde{\beta}^{2} \sigma^{2} \tilde{G}(a)\right]\right. \\
& -\frac{1}{2 d} \tilde{\beta}^{4} a^{2}\left[\sum_{j, i=1}^{\nu-2} \sigma_{j} \sigma_{i} \sum_{l \neq i, j}^{\nu-2} \Omega_{d} \frac{1}{d} \frac{\left(x_{l, j}\right)_{\alpha}\left(x_{l, i}\right)_{\alpha}}{\left|x_{l, j}\right|^{2}\left|x_{l, i}\right|^{2}}\left\{\begin{array}{l}
c_{2}^{2} \\
\frac{1}{4}(d-2)^{2} \tilde{G}\left(x_{l, j}\right) \tilde{G}\left(x_{l, i}\right)
\end{array}\right\}\right. \\
& \left.\left.+\sum_{j}^{\nu-2} \sigma_{j} \sum_{l \neq j}^{\nu-2} \sigma_{l} \Omega_{d} a^{-2} \tilde{G}\left(x_{l, j}\right)\left\{\begin{array}{l}
c_{2} \\
\frac{1}{2}(d-2) \tilde{G}(a)
\end{array}\right\}+\sum_{j}^{\nu-2} \sigma_{j}^{2} \frac{1}{2} \Omega_{d} a^{-2}(d-2) \tilde{G}^{2}(a)\right]<\sigma^{2}>\right]
\end{aligned}
$$

with

$$
<\sigma^{2}>=\sum_{\sigma \neq 0} \sigma^{2} w_{\sigma} w_{-\sigma} \exp \left[\frac{1}{2} \tilde{\beta}^{2} \sigma^{2} \tilde{G}(a)\right] .
$$

With the help of this relation, we can now write down the infinitesimal additional contribution to the partition function under the blocking transformation,

$$
\begin{gathered}
Z+\delta_{a} Z=\left(1+\Omega_{d} \frac{\delta a}{a} \frac{T V}{a^{d}} \sum_{\sigma \neq 0} w_{\sigma} w_{-\sigma} \exp \left[\frac{1}{2} \tilde{\beta}^{2} \sigma^{2} \tilde{G}(a)\right]\right) \sum_{\nu=2}^{\infty} \frac{1}{\nu !} \frac{\nu !}{(\nu-2) ! 2 !} \\
\int_{D_{0}} \frac{d^{d} x_{1}}{a^{d}} \cdots \int_{D_{\nu-3}^{\prime}} \frac{d^{d} x_{\nu-2}}{a^{d}}\left[\prod _ { j = 1 } ^ { \nu - 2 } \sum _ { \sigma _ { j } } w _ { \sigma _ { j } } \left(1-\frac{\delta a}{a} \frac{\Omega_{d}^{2}}{d} \sum_{\sigma \neq 0} w_{\sigma} w_{-\sigma} \exp \left[\frac{1}{2} \tilde{\beta}^{2} \sigma^{2} \tilde{G}(a)\right]\right.\right. \\
\left.\left.-\frac{d-2}{4 d} \tilde{\beta}^{4} \frac{\delta a}{a} \Omega_{d}^{2} \sigma_{j}^{2} \tilde{G}^{2}(a)<\sigma^{2}>\right)\right] \\
J^{[\nu-2]}\left(1-\tilde{\beta}^{4} \frac{\Omega_{d}^{2}}{d} \frac{\delta a}{a}\left\{\begin{array}{l}
-c_{2} \\
\frac{1}{2}(d-2) \tilde{G}(a)
\end{array}\right\} \frac{1}{2} \sum_{j}^{\nu-2} \sum_{l \neq j}^{\nu-2} \sigma_{j} \sigma_{l} \tilde{G}\left(x_{l, j}\right)<\sigma^{2}>\right) .
\end{gathered}
$$

Here, the first term leads to the renormalization of the free energy. The contribution of the third term on the r.h.s. of Eq. (A31) vanishes that can be seen after partial integration since $x_{l, i}$ and $x_{l, j}$ are not zero. The effects of all the other terms merge in the modifications of the parameters of the original partition function:

$$
\begin{aligned}
\delta_{a} \tilde{\beta}^{2} & =\tilde{\beta}^{4} \frac{\Omega_{d}^{2}}{d} \frac{\delta a}{a}\left\{\begin{array}{l}
-c_{2} \\
\frac{1}{2}(d-2) \tilde{G}(a)
\end{array}\right\}<\sigma^{2}> \\
\delta_{a} w_{\sigma} & =-\frac{\delta a}{a} w_{\sigma} \frac{\Omega_{d}^{2}}{d} \sum_{\sigma^{\prime} \neq 0} w_{\sigma^{\prime}} w_{-\sigma^{\prime}} \exp \left[\frac{1}{2} \tilde{\beta}^{2}{\sigma^{\prime}}^{2} \tilde{G}(a)\right]-\frac{d-2}{4 d} \tilde{\beta}^{4} w_{\sigma} \frac{\delta a}{a} \Omega_{d}^{2} \sigma^{2} \tilde{G}^{2}(a)<{\sigma^{\prime}}^{2}>.
\end{aligned}
$$

As we sum the various contributions to the changes of the parameters, we finally arrive at the flow equation (32), which completes its derivation. 


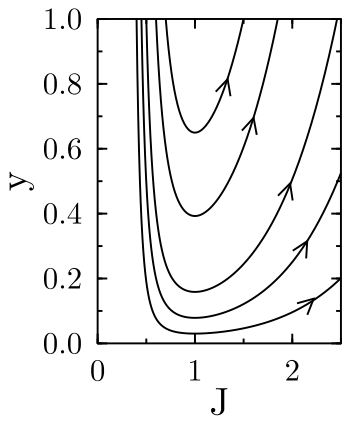

(a)

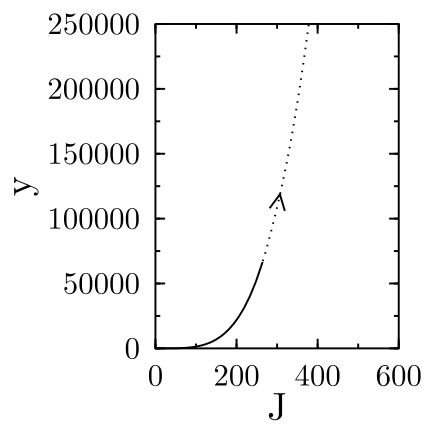

(b)

FIG. 1: RG flow for the three-dimensional GSGM, obtained by solving the WH-RG equations (a) Eqs. (21), (26), and (b) Eqs. (21), (23) outside the region of spinodal instability (solid line) and Eqs. 21), 25 inside the region of the spinodal instability (dotted line) by a fourth-order Runge-Kutta method. The RG trajectories are projected onto the subspace of the dimensionless coupling constants $y=\tilde{u}_{1}^{2}(k)$ and $J=1 / \tilde{\beta}^{2}(k)$. The lines represent the $\mathrm{RG}$ trajectories for various initial conditions, and the arrows indicate the direction of the RG flow.
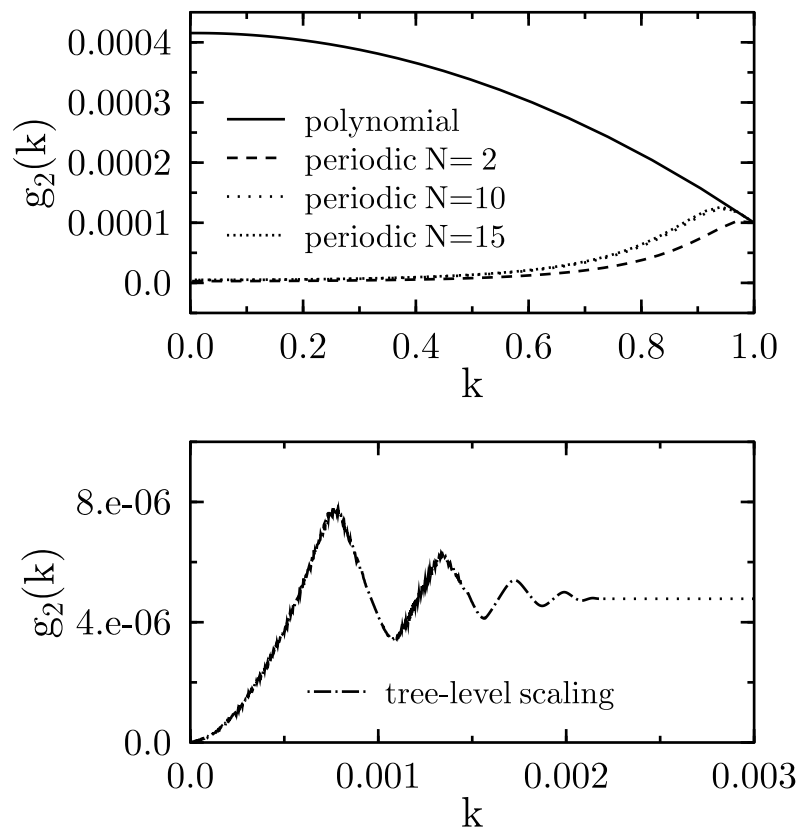

FIG. 2: RG-evolution of the dimensionful coupling constant $g_{2}(k)$ versus the running momentum cut-off $k$ (from $k=1 \ldots 0$ ) in $d=4$ [as a starting point for the RG flow, we fix $\tilde{\beta}^{2}(\Lambda=1)$ to the value $\left.128 \pi^{2}\right]$. Various truncations $N$ of the Fourier-expansion of the periodic potential have been employed. The upper and lower plots show the scale-dependence above and below the scale $k_{\mathrm{c}}$ of the spinodal instability which occurs at $k_{\mathrm{c}}=0.0022$ for $N=10$. The solution for the corresponding polynomial case is also indicated (solid line) [26, 39]. 

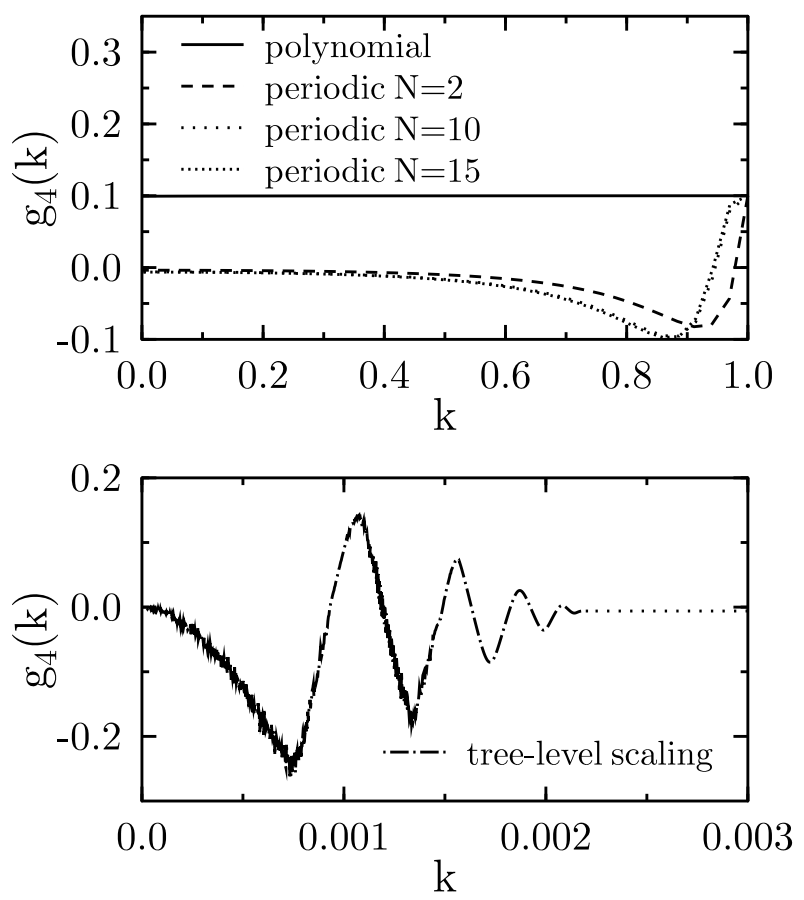

FIG. 3: The same as Fig. 2 for the coupling constant $g_{4}(k)$.
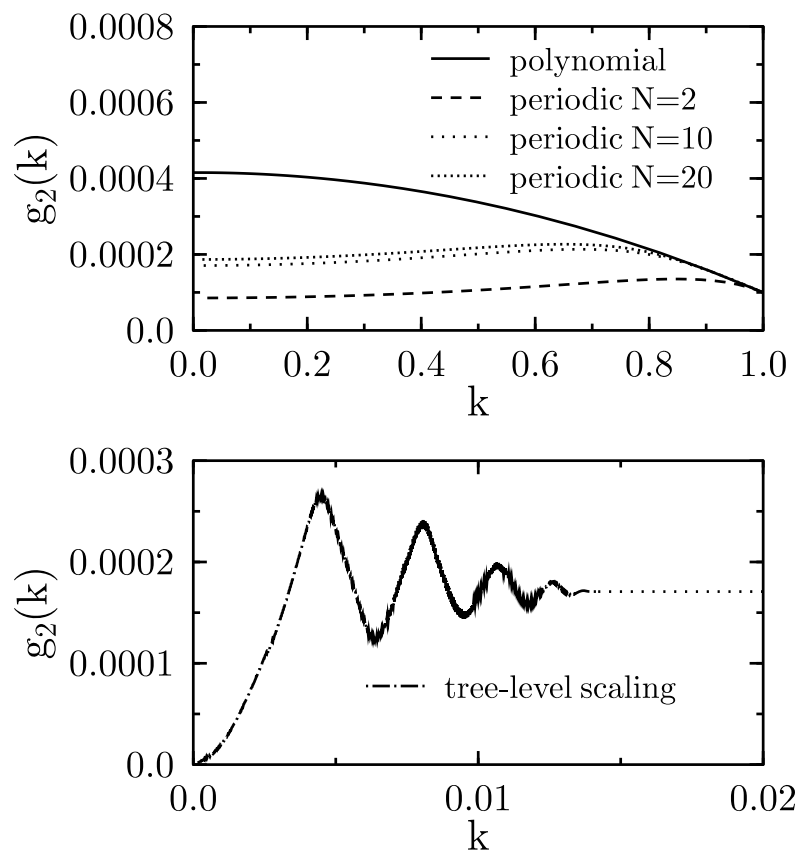

FIG. 4: The dimensionful coupling constant $g_{2}(k)$ is plotted versus the running momentum cut-off $k$ (from $k=1$ to 0 ) for $d=4$ dimensions, with $\tilde{\beta}^{2}(\Lambda=1)=32 \pi^{2}$. Various truncations $N$ of the Fourier-expansion of the periodic potential are employed. The upper and lower plots show, respectively the scale-dependence above and below the scale $k_{\mathrm{c}}$ of the spinodal instability which occurs at $k_{\mathrm{c}}=0.01413$ for $N=10$. The solution for the corresponding polynomial case is also indicated (solid line) [26, 39]. 

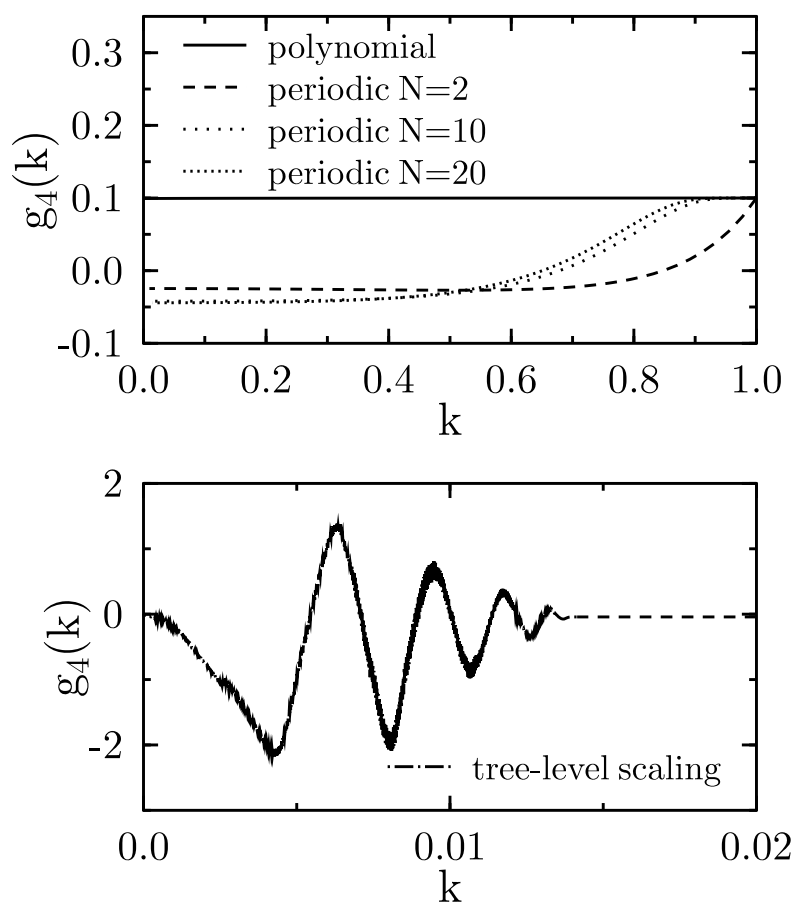

FIG. 5: The same as Fig. 4 for the coupling constant $g_{4}(k)$.

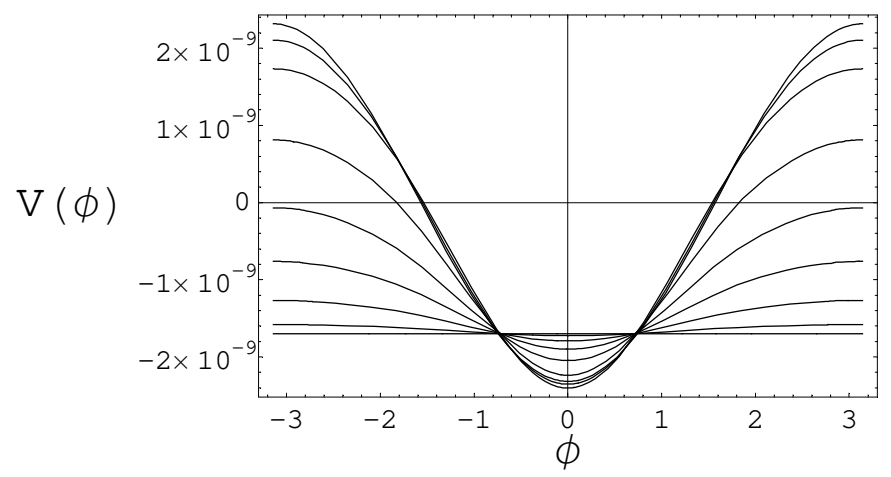

FIG. 6: The flattening of the dimensionful periodic potential $V_{k}(\phi)$ is plotted inside the region of spinodal instability, $k<k_{\mathrm{c}}$. The various curves with decreasing curvature at $\phi=0$ correspond to different values of the scale decreasing from $k=k_{\mathrm{c}}$ in steps of $\delta k=k_{\mathrm{c}} / 10$. 


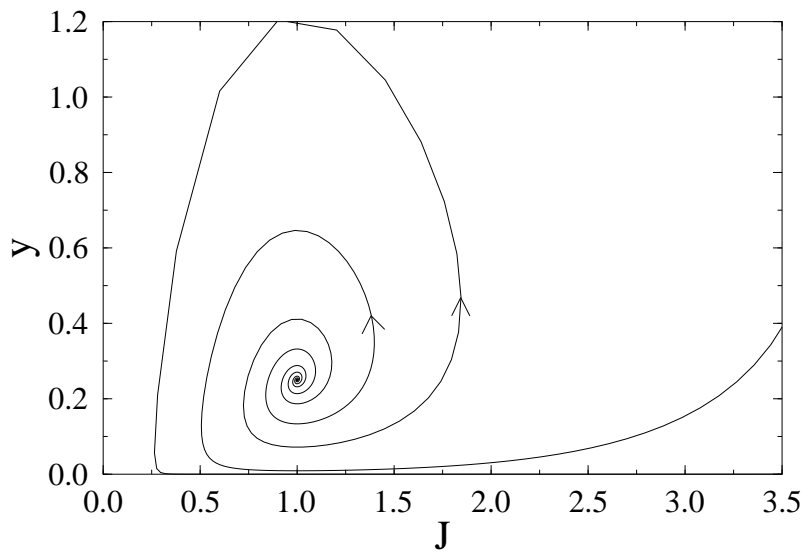

FIG. 7: RG flow for the ECG in the plane $(J, y)$, calculated without the terms of $\mathcal{O}\left(y^{2}\right)$ on the r.h.s. of Eq. (37b).

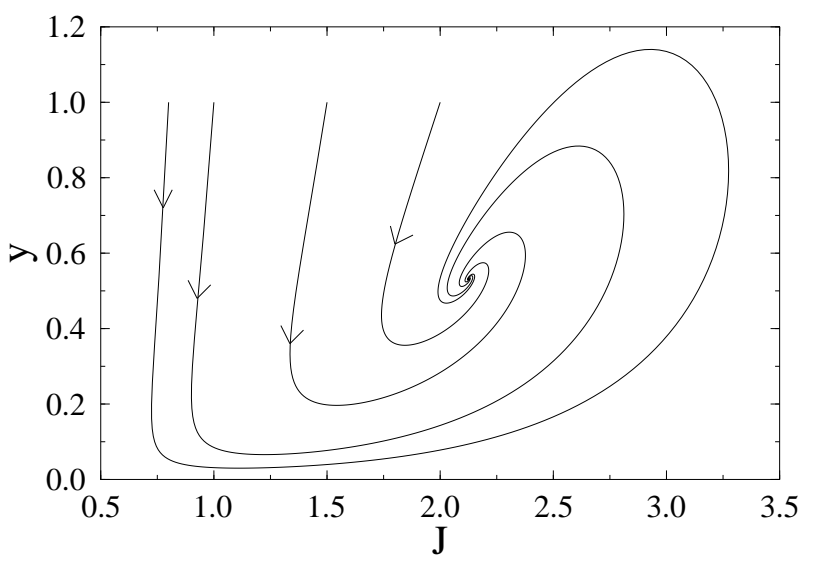

FIG. 8: RG flow for the ECG [Eq. [37] in the $(J, y)$-plane. 